\title{
Estudio de la utilización de los distintos servicios hos- pitalarios de pacientes nacidos en el extranjero en el Hospital General Universitario de Alicante HGUA (1 de enero de 2011-30 de junio 2014)
}

Study of utilization of different Hospital Services by foreign patients in Hospital General Universitario de Alicante (HGUA)

(1 st January 2011-30th June 2014)

\section{Estudo sobre a utilização de distintos serviços hospitalares no Hospital General Universitário de Alicante HGUA por pacientes estrangeiros} (1 de Janeiro de 2011-30 de Junho 2014)

Rosa Maria. Albert Sanchis ${ }^{1}$, Miguel Angel Fernández Molina ${ }^{2}$

${ }^{1}$ Enfermera. Doctora en Sociología; ${ }^{2}$ Enfermero. Doctor en Antropología

Cómo citar este artículo en edición digital: Albert Sanchis, R.Ma . y Fernández Molina, M.A. (2015). Estudio de la utilización de los distintos servicios hospitalarios de pacientes nacidos en el extranjero en el Hospital General Universitario de Alicante HGUA (1 de enero de 2011-30 de junio 2014). Cultura de los Cuidados (Edición digital), 19, 42. Disponible en: http://dx.doi.org/10.14198/ cuid.2015.42.10>

Correspondencia: C/Pintor Rodes $n^{\circ} 145^{\circ} \mathrm{C}$. CP. 03011 Alicante

Correo electrónico: albert_san@gva.es

Recibido: 16/01//2015; Aceptado: 11/06/2015

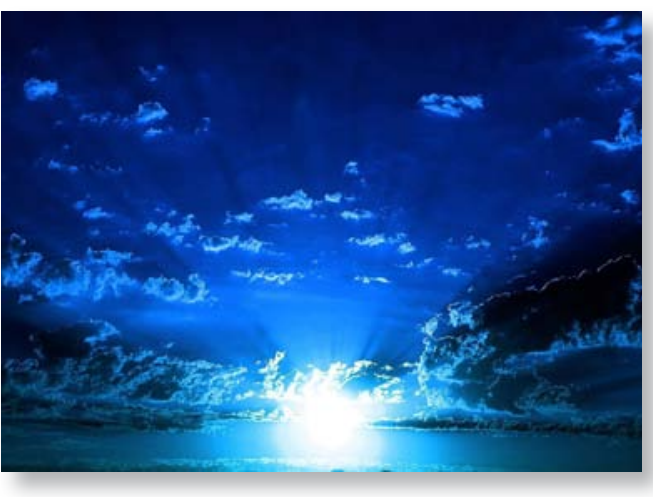

ABSTRACT

This article examines the importance of the current crisis and its impact on the health of the registered foreign population as a destabilizing demographic vector. It specifically examines the utilization by patients born abroad of different hospital services of the Hospital General Universitario de Alicante, considering the inter-group differences, which acquire, along with age, a decisive role with regard to the type of pathologies afflicting this group, and therefore, its cost in euros. The results obtained allow comparability over time and could be extrapolated to the rest of the Spanish territory.

Key words: cultural diversity, foreign, nationality, age, hospital services, province of Alicante, INE, HGUA, SNS, SIP, GRD, ODUSALUD, RDL 16/2012

\section{RESUMO}

Este artigo estuda a importância da crise atual como vector desestabilizador demográfico e sua incidência na saúde da população estrangeira inscrita nos serviços de saúde. Concretamente, o estudo faz uma análise da utilização dos distintos serviços hospitalares no Hospital General Universitário de Alicante, por parte dos pacientes 
estrangeiros, considerando as diferenças inter-grupal, que adquirem, junto com a idade, um papel decisivo no que se refere ao tipo de patologias que afligem este grupo e, portanto, seu custo em euros. Os resultados obtidos permitem a comparabilidade ao longo do tempo e podem ser extrapolados para o resto do território espanhol.

Palavras-chave: diversidade cultural, estrangeiros, nacionalidade, idade, serviços hospitalares, província de alicante, INE, HGUA, SNS, SIP, GRD, ODUSALUD, RDL 16/2012.

\section{RESUMEN}

Este artículo estudia la importancia de la crisis actual como vector desestabilizador demográfico y su incidencia en la salud de la población extranjera empadronada. Concretamente analiza la utilización por parte de los pacientes nacidos en el extranjero de los distintos servicios hospitalarios del Hospital General Universitario de Alicante, atendiendo a las diferencias intergrupales, que adquieren, junto a la edad, un papel determinante de cara al tipo de patologías que aquejan a este colectivo y su coste en euros. Los resultados obtenidos permiten su comparabilidad en el tiempo y podrían extrapolarse al resto del territorio español.

Palabras clave: diversidad cultural, extranjeros, nacionalidad, edad, servicios hospitalarios, provincia de Alicante, INE, HGUA, SNS, SIP, GRD, ODUSALUD, RDL 16/2012

\section{INTRODUCCIÓN}

Justificación teórica

Los profesionales sanitarios son conscientes de la diversidad cultural que conlleva el fenómeno de la inmigración así como de sus posibles repercusiones sanitarias (Fernández, 2006a); del mismo modo, los residentes extranjeros, generalmente personas mayores procedentes del centro y norte de Europa, también han sido objeto de atención por parte de las autoridades, lo que ha llevado a regular recientemente la asistencia sanitaria a la que tienen derecho, debido principalmente al alto coste de los tratamientos asociados a enfermedades crónicas y degenerativas típicas de la edad. El crecimiento de población extranjera en nuestro país y en la Comunitat Valenciana no solo se ha detenido, sino que está experimentando un notable descenso que ha llevado a preguntarnos si tiene su reflejo en el número de pacientes extranjeros ingresados y si este descenso en el padrón por sí solo justifica dicho descenso (nos preguntamos también si las medidas gubernamentales y autonómicas sobre el acceso a la asistencia sanitaria estarían afectando a este colectivo); en este sentido, se cuenta con una referencia en el tiempo y en un contexto concreto como es la situación minuciosamente descrita en el Hospital General Universitario de Alicante durante los años 2001 al 2004 (Fernández, 2006b) que podría ayudar a una mejor visualización e interpretación del fenómeno en un entorno globalizado pero contrastivo.

La crisis actual no sólo habría generado un éxodo de los ciudadanos con menos recursos económicos, sino que también habría provocado la salida masiva de aquellos otros inmigrantes cuya presencia en España se asocia al consumo (Mantecón, Huete \& Estévez, 2014). Otros autores, por el contrario, sostienen que durante la crisis no se ha producido una salida masiva de población extranjera y han aumentado el número de nacionalizaciones como única protección eficaz contra una política de inmigración inadecuada sobretodo de los países latinoamericanos (Melero et al., 2013: 14). El clima y el estilo de vida serían las princi- 
pales razones que inducirían a la emigración en el colectivo noreuropeo. No ha habido un éxodo masivo, debido a que la "huida" de este colectivo venía produciéndose desde hacía ya algunos años, aunque sería en 2014 cuando el INE ha actualizado el padrón y recogido estos cambios (Membrado, 2014). Otros informes recientes hablan de que en la provincia de Alicante están descendiendo las nacionalizaciones concedidas por residencia y se mantendrían al alza los permisos de residencia de larga duración (Barceló, 2014: 10).

En cuanto a la ciudad de Alicante, lugar donde se ubica el HGUA, Carlos Gómez Gil ha analizado sus diferentes barriadas y recoge, entre otras conclusiones, que entre los años 2008 y 2012 en 25 de los 42 barrios de la ciudad se ha producido un descenso de población, sobretodo de población autóctona, provocando importantes desequilibrios como el aumento de la desigualdad social a todos los niveles, que unido a una falta de interés y vigilancia del fenómeno por parte de las autoridades estaría impidiendo la anticipación a los principales problemas en los procesos de movilidad residencial (Gómez, 2014). Estos procesos de movilidad residencial podrían ser una consecuencia del trastorno por aculturación incluido en la Clasificación Internacional de Enfermedades DSM 5 como dificultad de aculturación'1. Ya en el año 2004 los residentes extranjeros se estaban alejando del país y preferían otros destinos para sus inversiones debido fundamentalmente al espectacular aumento de los precios de las viviendas y de la densidad espacial que indujo a una exacerbada sucesión residencial mayormente en las zonas de costa, empeorando la convivencia vecinal y aún más si cabe la falta o deficiencia de infraestructuras en numerosas urbanizaciones residenciales, sobretodo de las más alejadas del centro de las ciudades. Todo ello de enorme ca- lado en la aparición y agravamiento de procesos psicológicos asociados a ansiedad, estrés y depresión, que habrían empujado a este colectivo a reconsiderar su proyecto migratorio (Albert, 2007)

\section{MÉTODO}

Objetivos: El Objetivo general de este estudio consiste en analizar el uso de los Servicios/ Unidades de Hospitalización del HGUA de pacientes nacidos en el extranjero ingresados desde el 1 de Enero de 2011 hasta el 30 de Junio de 2014

\section{Objetivos específicos:}

- Describir la situación en el Estado Español, Comunidad Valenciana y Provincia de Alicante respecto a la evolución de la población extranjera, normativa legal y aspectos sanitarios de interés.

- Determinar Servicios/Unidades de Hospitalización con mayor número de ingresos de población nacida en el extranjero.

- Cuantificar los pacientes nacidos en el extranjero ingresados por servicios, con especial atención al número de mujeres extranjeras atendidas

- Cuantificar los pacientes nacidos en el extranjero por países

- Comparar resultados obtenidos en la investigación actual con resultados obtenidos en el estudio efectuado en el mismo Hospital durante el periodo 2001-2004.

- Identificar los principales GRDs de pacientes nacidos en el extranjero ingresados $y$ su coste.

Características del estudio descriptivo:

Población diana: pacientes nacidos en el extranjero ingresados en el Hospital General Universitario de Alicante 
Periodo del estudio: desde el 1 de Enero de 2011 hasta el 30 de junio de 2014.

Campo de estudio: Hospital General Universitario de Alicante (HGUA), elegido por dos razones fundamentales:

- Ubicación geográfica: situado en el litoral de la Costa Blanca, zona turística por excelencia y de innegable atractivo para muchos turistas y extranjeros residentes con orígenes culturales diversos.

- Tamaño: Es el segundo hospital más importante en la Comunitat Valenciana por $n^{\circ}$ de camas (815), después de "La Fe" en Valencia. La población en SIP en junio de 2014 es de 270.727 pacientes, siendo el hospital de referencia de toda la provincia de Alicante.

Unidades de estudio: Unidades de hospitalización y servicios del HGUA.

Instrumentos de recogida de datos: datos del Padrón Continuo a 1 de Enero de cada año durante el periodo 2008-2014, por dos razones fundamentales: porque la población extranjera empadronada reside en territorio español de forma estable al menos la mitad del año, y porque la población irregular en España no está en SIP.

El estudio de la población extranjera ingresada en el HGUA se ha fundamentado en el Conjunto Mínimo Básico de Datos (CMBD), datos en SIP, las principales conclusiones aportadas por el Observatorio del Derecho Universal a la Salud de la Comunitat Valenciana (ODUSALUD) y una revisión bibliográfica.

Análisis estadístico: EXCEL.

Para variables de distribución continua se estimó la media como índice de tendencia central y la desviación típica como índice de variabilidad. Los principales datos obtenidos se presentan en tablas, gráficos lineales, y de barras, tipo pirámide poblacional

\section{Evolución de la población extranjera (2008-} 2014)

España

La evolución de la población extranjera en España queda estrechamente ligada a la evolución de trabajadores afiliados a la Seguridad Social en alta laboral, teniendo en cuenta que en 1996 se produjo un cambio legislativo significativo que eliminó la distinción entre población de hecho y de derecho. Desde 1998 hasta 2008 el crecimiento poblacional es importante, debido en gran medida a los cinco procesos extraordinarios de regularización que tuvieron lugar entre los años 1998 y 2005, sobretodo al impacto del proceso extraordinario de normalización del año $2005^{2}$ contenido en la disposición transitoria tercera del R.D. 2393/2004, por el que se aprobó un nuevo Reglamento de Extranjería que otorgaba el permiso de residencia y trabajo a la existencia de un contrato ${ }^{3}$ de trabajo real con alta efectiva en la Seguridad Social, además de la ampliación de la adhesión de los países del Este de Europa a la Unión Europea en el año 2004 (Gómez, 2005).

Según datos INE definitivos, durante el periodo 2008-2013 la población total empadronada aumentó en 971.961 personas, al igual que la población extranjera empadronada (277.476), debido fundamentalmente a los ciudadanos de la UE27 que aumentaron en 256.717 personas. Por primera vez desde 1996 las cifras de la población extranjera total empadronada disminuyeron ligeramente en 2013 (-15.229 personas) debido a la bajada de los extranjeros no comunitarios, cuyo descenso se inició en 2011.

Tabla 1. Evolución de la población empadronada en España (2008-2013)

$\mathrm{Si}$ se tienen en cuenta los datos provisionales INE a 1 de Enero de 2014, esta tendencia 
a la baja se agudiza considerablemente registrando una variación interanual en términos absolutos de 545.980 extranjeros menos, de éstos, ahora son los extranjeros comunitarios quienes liderarían la caída con una variación interanual de 311.839 extranjeros menos; en términos relativos esta disminución sería del $57,1 \%$ para los extranjeros comunitarios y del $42,9 \%$ para el resto de extranjeros.

Tabla 2. Población empadronada en España en el año $2014^{\star} y$ su variación interanual 2013-2014*

Estos resultados, aunque hay que tomarlos con cautela por su provisionalidad, reflejarían un claro cambio de tendencia en España hacia una pérdida importante de población, tanto autóctona, como extranjera.

\section{Comunitat Valenciana}

En la Comunitat Valenciana se registraron 97.456 nuevos empadronamientos de extranjeros entre los años 1998-2001, cifra que contrasta con la del periodo de 2001-2005, con 375.162, casi cuatro veces más; Durante el periodo 1998-2005, 7 de cada 10 nuevos empadronamientos se debieron a población extranjera (Op.cit., 2005).

Según datos definitivos INE a 1 de Enero de cada año, en el periodo 2008-2013 tan solo se registraron 16.552 nuevos empadronamientos de extranjeros, alcanzándose en el año 2010 con 893.759 de extranjeros empadronados la cifra mayor (46.420 personas más que en 2008).

\section{Tabla 3. Evolución de la población extranjera empadronada en la Comunitat Valenciana.}

En 2009 se aprecia una ralentización, cuyo resultado se plasmaría en una variación interanual 2010-2011 con saldo negativo (-12.977 personas) que apenas se recupera en 2011$2012(+2.230$ personas) para nuevamente descender en 2012-2013 (-19.121 personas).

Tabla 4. Población empadronada en la Comunitat Valenciana en el año $2014^{*} y$ su variación interanual 2013-2014*

Si incorporamos los datos de 2014, aunque habría que tomarlos con cautela por su carácter provisional, la variación interanual 20132014 sería negativa para el total de población extranjera empadronada (-131.366 personas), rebasando casi 7 veces la cifra de la variación interanual anterior, siendo los ciudadanos de la Unión Europea los que arrastrarían esta caída (-106.743 personas: 81,2\%). La Comunitat Valenciana registra el mayor descenso en términos absolutos, muy por delante de la Comunidad de Madrid (-83.628) y Cataluña (-72.496), correspondiendo las menores reducciones de población extranjera a la ciudad autónoma de Ceuta (-132 personas), Principado de Asturias (-3.384) y Cantabria (-3.807). En términos relativos las mayores reducciones de población extranjera siguen dándose en la Comunitat Valenciana (-15,2\%); le siguen Aragón (-14,2\%) y Comunidad Foral de Navarra (-12,6\%). En la ciudad autónoma de Melilla el incremento relativo sería del 0,7\% (Notas de Prensa INE, 2014: 6)

\section{Provincia de Alicante}

Según datos definitivos INE a 1 de Enero de cada año, en el periodo 2008-2013 la población extranjera empadronada total de la provincia de Alicante en términos absolutos apenas aumentó en 23.049 personas, alcanzando en el año 2012 la cifra mayor (26.242 personas más que en 2008). Ya en 2008 se da una ralentización en el crecimiento de esta población obteniendo variaciones interanuales negativas 
en $2010-2011$ (-415 personas) y en 2012-2013 (-3.193 personas), llegando a su máximo exponente en 2013-2014, según datos provisionales, con una bajada muy significativa de 89.728 personas, y cualitativamente distinta de la que hasta ahora se estaba produciendo porque afectaría sobretodo a la población extranjera empadronada de la Unión Europea (-80.246: 89,4\%), casi 9 de cada 10 .

Tabla 5.Población empadronada en la provincia de Alicante en el año $2014^{\star} y$ su variación interanual 2013-2014*

Estas cifras, que no hay que olvidar tienen carácter provisional, marcarían un cambio brusco de ciclo, un antes y un después.

Por otra parte, se podría decir que durante el periodo 2008-2013 la ralentización en el crecimiento de población empadronada extranjera se debió fundamentalmente a la disminución de la población de los Países Americanos, y de éstos, fueron los países latinoamericanos los que lideraron la caída.

\section{Tabla 6. Población extranjera empadrona-} da en la provincia de Alicante según zona de procedencia.

Atendiendo a datos definitivos INE a 1 de Enero de 2013, la nacionalidad mayoritaria es la inglesa, que triplica holgadamente a la marroquí que sería la segunda, a muy poca distancia de la alemana y rumana, que al menos duplican a sus seguidores inmediatos. Si comparamos los datos definitivos de 2013 con los datos provisionales de 2014, tan solo dos nacionalidades, la rusa y la $\operatorname{argelina}^{4}$ ganarían población (1.460 y 1662 personas respectivamente). La nacionalidad británica con 39.254 personas menos es la que acusa un mayor descenso en términos absolutos por delante de la alemana que le seguiría (-14.475 perso-

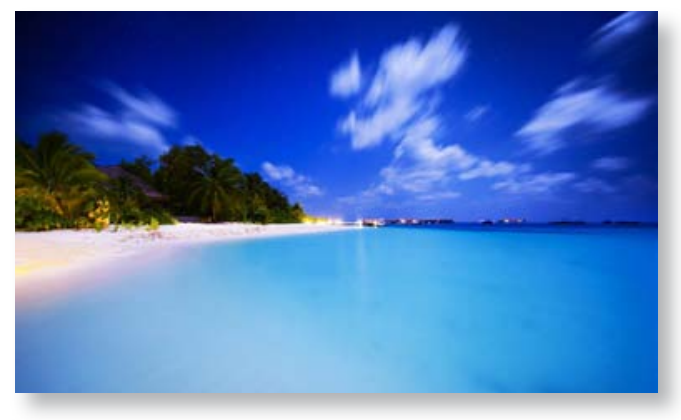

nas), ambas por muy por delante de la rumana (-3.833 personas) y la holandesa ( -3.543 personas) que serían los siguientes; pero en términos relativos es la nacionalidad alemana la que más desciende $(-40,3 \%)$, por delante de la británica $(-30,1 \%)$, que a pesar de todo seguiría siendo la nacionalidad mayoritaria (todos estos resultados deberán ser tomados con la cautela que se requiere por ser provisionales).

\section{Tabla 7. Población extranjera empadronada} en la provincia de Alicante según nacionalidad (doce primeras)

Enlazando de nuevo con los datos definitivos INE del Padrón Continuo a 1 de Enero de 2013 la población total es de 1.945 .642 personas, de las cuales, $469.417(24,1 \%)$ son extranjeros empadronados (51,4\% hombres y $48,6 \%$ mujeres).

Figura 1. Población extranjera empadronada por edad (grupos quinquenales) y sexo en la provincia de Alicante. Datos INE definitivos a 1 de Enero de 2013.

Así pues, y a pesar de que en las franjas de edades de 20-29 años, 55-64 años y a partir de los 90 años predomina el sexo femenino, la razón de sexo está inclinada hacia el sexo masculino (por cada 100 mujeres hay aproximadamente 106 hombres) siendo las franjas de edades comprendidas entre los 30-49 años y los 70-89 años las que más aglutinarían esta mayoría masculina. 
Tabla 8. Población extranjera empadronada por edad (grupos quinquenales) y sexo en la provincia de Alicante.

Se trata mayoritariamente de una población adulta joven, siendo la franja de edad comprendida entre los 25-44 años la que aunaría esta mayoría (30,1\%); la franja de edad comprendida entre los 55-74 años, con un 29,6\% no es nada desdeñable, tratándose una población adulta mayor que por su idiosincrasia podría precisar con asiduidad de diferentes servicios básicos de la sociedad española, como es el Sistema Nacional de Salud (SNS), al igual que las familias con hijos en edad escolar; además, por razones obvias a partir de los 75 años (8\%) la probabilidad de enfermar o el agravamiento de las patologías previas aumenta, por lo que la frecuentación de los servicios de salud sería muy alta en este segmento poblacional.

Dicho queda el importante declive de la población extranjera empadronada, tanto en España, como en la Comunitat Valenciana según los datos provisionales INE a 1 de Enero de 2014; la provincia de Alicante tampoco escaparía a esta realidad, arrojando la variación interanual 2013-2014 cifras negativas en todos los grupos quinquenales y en ambos sexos, con excepción de los centenarios.

Tabla 9. Variación interanual 2013-2014 de la población extranjera empadronada por edad (grupos quinquenales) y sexo en la provincia de Alicante.

En términos relativos tan solo la franja de edad de 65-79 años acumularía 1/4 parte del descenso total $(-25,7 \%)$, de éstos, es el grupo quinquenal de 65-69 años el que perdería más población $(-10,1 \%)$. De manera importante acusarían también este descenso las franjas de edades entre 30-49 años (-22,6\%) y 50-64 años (-21\%), muy por delante de las franjas de edades de 0-14 años (-6,2\%), 1529 años $(-14,8 \%)$ y los mayores de 80 años $(9,5 \%)$. También se daría un importante descenso en las mujeres de edad fértil de 15 a 49 años $(-32,1 \%)$, siendo la franja de edad de 25-34 años la que más acusaría este descenso $(-12,7 \%)$; a pesar de ello la población masculina registraría un mayor descenso poblacional $(-51,8 \%)$, siempre recordando el carácter provisional de estos datos.

Figura 2. Población extranjera empadronada por edad (grupos quinquenales) y sexo en la provincia de Alicante. Datos INE provisionales a 1 de Enero de 2014.

\section{Legislación}

Europea

Se alude ahora, por su importancia, a las principales leyes que regulan el derecho a la sanidad pública de la población extranjera, como la Declaración Universal de los Derechos Humanos (1948), que en línea con el preámbulo de la Constitución de la Organización Mundial de la Salud (1946) y el Pacto Internacional sobre Derechos Económicos, Sociales y Culturales (1966), ratificado por España el 13 de Abril de 1977, reconoce el derecho de toda persona al disfrute del más alto nivel de su salud física y mental.

En la Unión Europea cada país es responsable de definir el sistema de derechos y obligaciones en materia de Seguridad Social que considere más oportuno, en este sentido, el Reglamento del Consejo 118/97, modifica y actualiza el Reglamento CEE 1408/71 por el que se regula la aplicación de los sistemas de Seguridad Social a los trabajadores y a los miembros de sus familias para sus desplazamientos dentro del ámbito comunitario. 
Española

En España la asistencia sanitaria a extranjeros es un derecho que queda establecido en el artículo 43 de la Constitución Española, regulado por la Ley 14/1986 General de Sanidad, el Real Decreto 1088/1989 que extiende la cobertura a las personas sin recursos económicos suficientes que tengan establecida su residencia en territorio nacional y no tengan derecho a la asistencia sanitaria por otra vía y la Ley Orgánica 8/2000, de 22 de Diciembre que modifica la Ley de Extranjería 4/2000 de 11 de Enero, sobre derechos y libertades de los extranjeros en España y su integración social, extendiendo la condición de asegurado a quienes acrediten no superar unos ingresos que son determinados reglamentariamente, $y$ a algunos vínculos de parentesco: cónyuge, ex cónyuge a cargo del asegurado, descendientes menores de 26 años o que tengan una discapacidad igual o mayor al 65\%.

Todas estas leyes supusieron enormes avances en materia de sanidad pública que se han visto afectados con la entrada en vigor del RDL 1192/2012, que regula, entre otros, la condición de asegurado y de beneficiario a efectos de la asistencia sanitaria en España con cargos a fondos públicos a través del Sistema Nacional de Salud y del RDL 16/2012 de medidas urgentes para garantizar la sostenibilidad del Sistema Nacional de Salud y mejorar la calidad y seguridad de sus prestaciones, ostentando dicha "condición de asegurado" los trabajadores afiliados a la Seguridad Social, los pensionistas, las personas en paro con subsidio o que hayan agotado la prestación por desempleo y figuren inscritos como demandantes de empleo, y los residentes extranjeros legales; no reconociéndose la condición de asegurado a los extranjeros que no estén registrados ni tengan autorización de residencia con indepen- dencia de sus ingresos, lo que afectaría tanto a turistas como a residentes de facto, es decir, a los residentes extranjeros que se encuentren en situación administrativa irregular ${ }^{5}$.

\section{Comunitat Valenciana}

Por su parte, la Ley 16/2003, de 28 de Mayo, de Cohesión y Calidad del Sistema Nacional de Salud, establece en su artículo 3 la titularidad de los derechos a la protección de la salud y a la atención sanitaria que se concreta en la Comunitat Valenciana en su propia normativa: el Decreto 26/2000, de 22 de Febrero que estableció el derecho a la asistencia sanitaria a ciudadanos extranjeros y creó la tarjeta solidaria enmarcando los derechos establecidos previamente en la Ley Orgánica 4/2000 y ampliando la cobertura sanitaria a los extranjeros no empadronados y carentes de recursos.

El pleno del Gobierno Valenciano aprobó el 12 de septiembre de 2014 el Proyecto de ley de la nueva Ley de Salud de la Comunitat Valenciana, que define el Sistema Valenciano de Salud como organización y establece las prestaciones sanitarias a las que pueden acogerse los valencianos refundiendo seis leyes, un decreto legislativo y cuatro decretos (LevanteEMV, 2014); de ser aprobada, esta ley podría permitir que en un futuro sea la propia Consellería de Sanitat la que a través de Reglamentos pueda modificar aspectos importantes del sistema sanitario valenciano sin previo paso por las Cortes Valencianas (SATSE, 2014).

\section{Aplicación de los RDL 1192/2012 y 16/2012}

La aplicación de los RDL 16/2012 y $1192 / 2012$ no está siendo igual en todas las Comunidades Autónomas, y esta inequidad está creando diferencias importantes en el acceso al diagnóstico y tratamiento en los colectivos más desfavorecidos; en este sentido, el 
Observatorio del Derecho Universal a la Salud de la Comunitat Valenciana (ODUSALUD) vela por el cumplimiento del Derecho a la Salud a través de la ciudadanía que colabora con las entidades participantes. Desde su creación en Noviembre de 2012 este Observatorio ha recibido 718 notificaciones, 120 en el segundo trimestre de 2014, apreciándose un incremento que se justificaría por la mayor participación de las entidades colaborativas, que empezaron siendo tres y ahora son casi setenta.

Aspectos más destacables de las notificaciones recibidas:

- 79 casos de menores desatendidos

- Exigencia de compromiso de pago previo a la asistencia y la facturación (29\%)

- Un $14 \%$ tendría dificultades económicas de acceso a medicamentos

- Trabas administrativas: dos de cada tres casos no llegan a ser atendidos por personal sanitario

- Una de cada cuatro incidencias afecta a ciudadanos de la Unión Europea. El 35\% afecta a personas latinoamericanas. África subsahariana (16\%) y el Magreb (10\%) son los siguientes

- El 55\% de las incidencias afecta a mujeres

- El 86\% de los registros procede de las capitales de provincia (Alicante: 38\%, Castellón: 4\% y Valencia: 44\%)

- El 17\% de las incidencias se produce en hospitales, sobretodo en el área de urgencias; $74 \%$ tiene lugar en los centros de salud

Este Observatorio también ha notificado que el Programa Valenciano de Protección de la Salud implantado carece de una estrategia informativa adecuada, por lo que se limitan las posibilidades de mejorar el acceso a la asisten- cia sanitaria de las personas excluidas, y sigue instando a la administración sanitaria a que asuma todas las responsabilidades y ponga en marcha medidas adecuadas para verificar $y$ garantizar con especial atención este derecho universal en los grupos más vulnerables.

\section{El Sistema de Información Poblacional de la}

\section{Generalitat Valenciana (SIP)}

El SIP se crea en 1999 por el Decreto 126/1999, de 16 de agosto, del Gobierno Valenciano, con el objetivo de mejorar la relación de la ciudadanía con la administración, ser de utilidad en la planificación, gestión administrativa, ordenación de recursos sanitarios y servir de soporte para la gestión de la tarjeta sanitaria individual SIP $^{6}$.

Durante el periodo 2005-2012 hubo un aumento en términos absolutos de 256.267 personas extranjeras registradas en SIP, que supone un aumento porcentual de 4,6\% Esta población empieza a descender en términos absolutos a partir del año 2009, al igual que la población total de alta en SIP, no así en términos relativos, que lo hizo a partir de 2010, y desde entonces sigue esta tendencia a la baja.

Tabla 10. Evolución de la población total y extranjera registradas en SIP en la Comunitat Valenciana (2005-2012)

Los extranjeros de alta en SIP se agrupan en distintas modalidades organizadas en base a la Ley 6/2008 de 2 de junio de la Generalitat, de Aseguramiento ${ }^{7}$ Sanitario del Sistema Sanitario Público de la Comunitat Valenciana. Según el Informe mensual de Junio de 2014 del SIP, el total de población en SIP de la Comunitat Valenciana era de 5.067.467 personas, concentrándose la mayoría de ellas $(93,4 \%)$ en el grupo de asegurados INSS-TSE. Los extranjeros en SIP sumarían un total de 740.262 per- 
sonas y tan solo en el primer semestre de 2014 esta población disminuyó en 25.717 personas, siendo aún mayor este descenso si observamos otras modalidades, pues tan solo en el grupo Sin cobertura/Mutualismo privado/No acreditado fue de 29.585 extranjeros menos. En los grupos de asegurados INSS-TSE y de cobertura SNS-Consellería esta población aumenta en 2.576 y 2.046 personas respectivamente (Generalitat Valenciana, 2014a)

Tabla 11. Modalidades de acreditación de la asistencia sanitaria. Evolución de los ciudadanos extranjeros en SIP en el primer semestre de 2014.

El descenso poblacional en SIP es mucho mayor que su ganancia poblacional, pues a pesar de que en el primer semestre de 2014 han habido 4.622 nuevas incorporaciones de población extranjera en SIP, esta cifra negativa (-25.717) supera en más de cinco veces la cifra de incorporaciones.

\section{PRESENTACIÓN DE RESULTADOS}

En el año 2010 el Departamento de Salud Alicante. Hospital General atendía una población total de 290.238 personas, de las cuales 40.949 (14,1\%) eran extranjeras (Ortiz. et al., 2011: 44); en Junio de 2014 la población total atendida era de 270.727 personas, de las cuales 29.854 (11\%) son extranjeras (Op. cit., 2014a). El Hospital General Universitario de Alicante (HGUA) es también centro de referencia de atención para toda la provincia de Alicante en diversas especialidades, por lo que la población asignada es mucho mayor. Entre el 1 de Enero de 2011 y el 31 de Diciembre de 2013 se registraron en el HGUA un total de 9.343 ingresos de pacientes nacidos en el extranjero, con una edad media de 44,7 años y 116 nacionalidades. Aproximadamente 3 de cada 4 de estos pacien- tes pertenecen al ámbito europeo y americano; los Países Africanos copan casi todo el resto seguidos muy de lejos por Asia y Oceanía. La distribución por sexos, es aproximadamente de 60/40, claramente sesgada hacia el sexo femenino (5.475: 57,9\% mujeres/3.868: 42,1\% hombres).

Tabla 12. Porcentaje de población extranjera ingresada en el HGUA según zona de procedencia.

En general, la tendencia actual es hacia una disminución progresiva del número total de ingresos de esta población.

Tabla 13. Población nacida en el extranjero ingresada en el HGUA desde el 1 de Enero de 2011 hasta el 31 de Diciembre de 2013 y sus respectivas variaciones interanuales.

Si se incorporan los datos del primer semestre de 2014 y se comparan con los del primer semestre de 2013, los usuarios autóctonos aumentaron en 418 personas (2,9\%), debido fundamentalmente a la población femenina $(2,8 \%)$, mientras que los nacidos en el extranjero descienden $(-3,4 \%)$ debido a su población masculina (-5\%), ya que se da un aumento en esta población femenina (1,6\%); el porcentaje aproximado de casos perdidos sería de $0,7 \%$

Tabla 14 Población nacida en el extranjero ingresada en el HGUA desde el 1 de Enero hasta el 30 de Junio (años 2013 y 2014). Variación intersemestral.

Población nacida en el extranjero ingresada en el HGUA. Unidades de ingreso

El servicio con mayor número de ingresos de pacientes nacidos en el extranjero fue obstetricia, 21,6\% del total de los ingresos de pacientes nacidos en el extranjero, a gran dis- 
tancia del siguiente servicio, cirugía general $(7,1 \%)$, que a su vez se distancia en más de dos puntos porcentuales con el servicio de cardiología (4,6\%), la Unidad de Corta Estancia $(4,5 \%)$ y Neurocirugía $(4,3 \%)$ y con más de tres puntos porcentuales con los servicios de ginecología $(3,4 \%)$, cirugía ortopédica y traumatológica $(3,4 \%)$, neurocirugía $(3,3 \%)$ y la unidad hepática $(3,1 \%)$, agrandándose la distancia porcentual con el resto de servicios según va disminuyendo el número de pacientes extranjeros ingresados en éstos.

Tabla 15. No pacientes nacidos en el extranjero ingresados en el HGUA, desglosados por códigos ${ }^{8}$ de servicios médicos.

El área de obstetricia junto al área ginecológica suma aproximadamente $1 / 4$ de los ingresos totales de pacientes nacidos en el extranjero, lo que explicaría el predominio del sexo femenino en cuanto al número de ingresos de pacientes extranjeros totales en el HGUA que contrasta con el hecho de que la pirámide poblacional de la población extranjera en la provincia de Alicante esté sesgada hacia los hombres (según datos INE provisionales a 1 de Enero de 2014: 51,4\% para los hombres y $48,6 \%$ para las mujeres).

\section{Población nacida en el extranjero ingresada} en el HGUA. Países de nacimiento

Observando la tabla $n^{\circ} 16$, los primeros 12 países reúnen casi el $70 \%$ de los ingresos totales de extranjeros en el HGUA, copando solo los 6 primeros más de la mitad de éstos (51\%) y tan sólo los 2 primeros el 22,3\%

Tabla 16. Primeros doce países con mayor número de ingresos totales en el HGUA (2011-2013)

Marruecos con 1.244 pacientes ocupa el primer lugar en cuanto a número de ingresos totales de extranjeros (13,3\%), Argelia (5,3\%) es el segundo país africano a destacar. Los países americanos con Colombia (9\%), Ecuador (7,8\%) y Argentina (6,5\%) ocuparían los primeros lugares con más de 600 ingresos cada año. En cuanto a los países europeos, Reino Unido ocupa el primer lugar con un 7,5\% de la totalidad de ingresos en este periodo, seguido muy de cerca por Francia $(6,9 \%)$ y Rumanía $(4,6 \%)$.

Marruecos lidera un mayor número de servicios como cirugía general (13\%), cardiología $(16,6 \%)$, la unidad de corta estancia (16,9\%), urología $(16,1 \%)$, neurología $(10,3 \%)$ o cirugía ortopédica y traumatológica $(15,7 \%)$. El país europeo que lidera un mayor número de servicios como neurocirugía (18,3\%), cirugía vascular $(23,4 \%)$, o la unidad hepática $(13,2 \%)$ es el Reino Unido; Francia es el segundo país europeo ocupando el quinto lugar, liderando también algunos servicios como neumología $(12,4 \%)$ y oncología $(13,7 \%)$. Con respecto al área de ginecología, son Colombia (15,9\%), Marruecos y Ecuador (9\%) quienes ostentan el mayor número de ingresos, le siguen Rumanía $(8,4 \%)$, Francia (5,3\%), Argentina $(4,7 \%)$ y Bolivia $(4,4 \%)$

Al incorporar los datos semestrales (20132014), de los doce países con mayor número de ingresos, la mayoría registrarían una disminución en sus cifras, tanto en términos absolutos como en términos relativos.

Tabla 17. Variación intersemestral desde el 1 Enero al 30 Junio (años 2013 y 2014) de la población nacida en el extranjero ingresada en el HGUA, según países.

Dos países latinoamericanos, Ecuador y Colombia (-15,3\% y $-12,3 \%$ respectivamente) son los que registrarían el mayor descenso. Alemania $(-8,8 \%)$ es el primer país europeo 


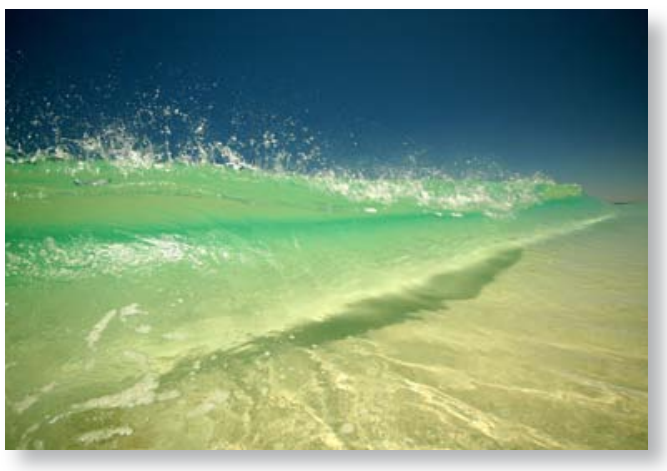

seguido por Reino Unido $(-5,8 \%)$ y Francia (-1,9\%); Argelia (-4,8\%) y Marruecos (-4,3\%) también acusarían importantes descensos. Por otro lado, es un país europeo, Rumanía $(14,9 \%)$ quien registra la mayor subida junto a Argentina (14,5\%). Otros países, aunque con menor número de ingresos, también han registrado importantes aumentos, como es el caso de Letonia, que no registró ningún ingreso en el primer semestre de 2013 y en 2014 registra 10 ingresos.

Población nacida en el extranjero ingresada en el Servicio de Obstetricia del HGUA. Principales resultados

El área obstétrica merece especial atención, porque inclina poderosamente la razón de sexo hacia las mujeres e identifica, entre otras variables, el porcentaje de partos de las pacientes nacidas en el extranjero, con respecto a las autóctonas.

Desde el 1 de Enero de 2011 hasta el 31 de Diciembre de 2013 se atendieron un total de 9.375 mujeres, de las cuales, el 21,2\% fueron mujeres nacidas en el extranjero (2.020 mujeres).

Tabla 18. Población nacida en el extranjero atendida en el servicio de Obstetricia del HGUA desde el 1 de Enero de 2011 hasta el 31 de Diciembre de 2013 y sus respectivas variaciones interanuales.
Durante este periodo se registró un importante descenso en la totalidad del número de ingresos tanto en términos absolutos (-635 personas), como en términos relativos $(-6,8 \%)$ que confirmaría una tendencia a la baja tanto en la población autóctona (-458 personas) como en la población de mujeres nacidas en el extranjero (-169 personas), con un 6,3\% y $8,3 \%$ respectivamente.

Gráfico 1. Variaciones interanuales e intersemestrales de mujeres nacidas en el extranjero ingresadas en el servicio de obstetricia del HGUA (2011-2014)

Si se incorporan los datos del primer semestre de 2014, la población ingresada total disminuye un $6,3 \%$, pero es la población autóctona la que registra una mayor caída $(-9,7 \%)$ frente a la población nacida en el extranjero $(-8,3 \%)$. El porcentaje de mujeres nacidas en el extranjero que fueron atendidas en el primer semestre de 2014 apenas disminuye un 0,5\% con respecto al periodo 2011-2013.

Cabría considerar también a los países con mayor número de ingresos, siendo Marruecos el país que cuenta con más ingresos (340: $16,8 \%$ ), seguido a considerable distancia por Ecuador (212: 10,5\%), Colombia (180: 8,9\%), Argelia (144: 7,1\%), Rumanía (140: 6,9\%), Argentina (117: 5,8\%) y el resto de nacionalidades. El primer país africano es Nigeria que ocuparía el octavo lugar $(63: 3,1 \%)$ y China con el décimo, sería el primer país asiático (52: 2,6\%). En el onceavo lugar aparece Francia (47: 2,3\%), el primer país europeo, a gran distancia de Alemania (22: 1,1\%), Bulgaria y Reino Unido (18: 0,9\%) que serían los siguientes y ocuparían el veintidosavo, veinticincoavo $\mathrm{y}$ veintiseisavo lugares respectivamente.

La edad media de los ingresos totales es de 31,7 años; siendo la edad media de la pobla- 
ción española 31,5 años y la de la población extranjera 30,8 años. Las pacientes nacidas en Rumanía son las más jóvenes (28,3 años). El país con mayor edad media es Francia con 35,3 años.

Al incorporar los datos semestrales de 2013 y 2014 esta tendencia al descenso se confirmaría.

Tabla 19 Variación intersemestral (1 Enero-30 Junio 2013 y 2014) de la población ingresada en la unidad de obstetricia del HGUA.

Los seis primeros países se mantendrían, pero se daría un importante aumento de la Federación Rusa (3,7\%), que del duodécimo lugar, pasaría al séptimo y China $(3,3 \%)$ que ocuparía el octavo. El primer país africano sigue siendo Nigeria que bajaría una posición $(2,9 \%)$. Francia $(2,1 \%)$, el primer país europeo, también baja una posición, y tanto Alemania como Reino Unido no registran ningún ingreso; Polonia con dos ingresos, y Bélgica, Bulgaria y Letonia con uno, serían los únicos países europeos que contarían con ingresos en este semestre.

En cuanto a los diagnósticos, de un total de 39 diagnósticos obstétricos, es el parto vaginal sin complicaciones el diagnóstico con mayor número de casos, 3.090 pacientes, de las cuales 691 son nacidas en el extranjero $(7,4 \%)$, le sigue con 2.370 pacientes, 463 extranjeras $(4,9 \%)$ el parto vaginal con complicaciones $y$ la cesárea sin complicaciones; de un total de 1.016 cesáreas, $215(2,3 \%)$ se practicaron a mujeres nacidas en el extranjero.

\section{Gasto sanitario de los diagnósticos principa-} les de los pacientes nacidos en el extranjero atendiendo a los GRDs.

El gasto sanitario que ocasiona la atención sanitaria a los pacientes no autóctonos es im- portante tenerlo en cuenta, para ello, los GRDs (Grupos Relacionados por el Diagnóstico) son una herramienta de gestión normalizadora, en la que utilizando un programa informático, alimentado con los datos de los pacientes dados de alta hospitalaria, que forman parte del Conjunto Mínimo Básico de Datos (CMBD), podemos clasificar a los pacientes en grupos clínicamente similares y con consumo de recursos sanitarios parecidos. En este epígrafe, y a título informativo, se muestran los 15 códigos GRDs más frecuentes y su descripción, atendiendo al número de pacientes extranjeros ingresados, y el coste que cada caso reportaría, según la Ley de Tasas (ejercicio 2014).

Tabla 20. Diagnósticos principales de los pacientes nacidos en el extranjero ingresados en el HGUA (2011-2013) atendiendo a GRDs, y su coste (en euros)

\section{DISCUSIÓN}

Entre el 1 de Enero de 2001 y el 31 de diciembre de 2004 hubo un total de 147.522 ingresos de pacientes en el HGUA, de los cuales $9.792(6,6 \%)$ correspondieron a pacientes extranjeros de 91 nacionalidades diferentes (teniendo en cuenta que un mismo paciente puede tener múltiples ingresos). Concretamente, en el año 2004, las personas nacidas en el extranjero supusieron en términos relativos el 7,7\% del total de ingresos, con una edad media de 42,7 años y una desviación típica de 21,8 . Por otra parte, durante el periodo 20112013 la edad media fue de 44,7 años con una desviación típica de 19,9 y un ligero aumento de la diversidad cultural (un total de 106 nacionalidades); si observamos la evolución de la edad media del colectivo en este periodo, en 2011 fue de 43,9 años; en 2012 de 45,1 años y en 2013 de 45,4 años, por lo que la tendencia 
es hacia un aumento de la edad media de los ingresos totales, dándose la circunstancia de que el aumento global durante todo el periodo 2004-2013 es de 2,7 años, pero tan solo la variación interanual 2011-2012 fue de 1,2 años, casi la mitad del aumento que se dio durante todo este periodo. Este dato no puede pasar desapercibido y se ha indagado en los últimos datos del Sistema Básico de Vigilancia ${ }^{9}$ de la Comunitat Valenciana (Informe Marzo, 2014), no encontrando ningún aumento significativo en lo que se refiere a la casuística de enfermedades de declaración obligatoria; también se consultó con el Dr. Torrús, médico adjunto de la Unidad de Enfermedades Infecciosas y responsable de la Consulta de Enfermedades Importadas y Parasitología del HGUA, no encontrando ningún cambio significativo él tampoco.

Al hilo de este dato, durante el innegable periodo de crisis que atravesamos estamos asistiendo a un descenso importante de población extranjera en nuestro territorio que podría deberse en gran parte al efecto combinado de la emigración y de la adquisición de nacionalidad española (INE Notas de prensa, 2014b). Las medidas restrictivas de acceso a la sanidad pública dificultan sobretodo el acceso a la misma de población extranjera joven al retirarles la tarjeta sanitaria impidiendo su atención normalizada, lo que ha podido influir en que los pacientes extranjeros que hacen uso de los servicios de ésta tengan mayor edad, debido a que llevan más tiempo en el país, tengan doble nacionalidad u otras situaciones que les permiten este acceso (este punto fue consultado con el especialista en migraciones y profesor de la Universidad de Alicante Carlos Gómez Gil).

La crisis también ha tenido un impacto profundo en los residentes europeos funda- mentalmente por la pérdida de poder adquisitivo junto a la histórica subida de impuestos como el IRPF y la obligación de declarar sus bienes radicados en el extranjero a Hacienda que han elevado los costes de residir en España y espantado a muchos de ellos (así lo demostrarían las cifras INE a 1 de enero de 2014, que aunque con carácter de provisionalidad, estarían marcando un cambio muy brusco de tendencia). Nacionalidades como la británica o la alemana, además, se han visto especialmente afectadas debido fundamentalmente a una significativa devaluación de la libra esterlina, la británica, y a que pagan menos impuestos que en España debido a importantes deducciones fiscales, la alemana (Llamas, 2014).

\section{CONCLUSIONES}

Según datos INE definitivos, durante el periodo 2005-2013 la provincia de Alicante registró un aumento en términos absolutos de 157.777 personas extranjeras, que supone un aumento porcentual de 5,6\% a pesar del azote de la crisis. El año 2013 es el primer año que la población extranjera disminuye en esta provincia (-3.193 personas) debido fundamentalmente a los países latinoamericanos. Los datos INE de 2014, si bien tomados con cautela por su carácter de provisionalidad, serían demoledores en cuanto al descenso de población extranjera, pero ya no son los países latinoamericanos los que lideran esta espectacular caída, siendo ahora la Unión Europea (53,1\% del total de los extranjeros empadronados) quien marcaría un brusco cambio de tendencia. Este éxodo sin precedentes dejaría en la provincia de Alicante el padrón por debajo de 1,9 millones de personas a pesar del ligero incremento del número de nacionales (6.645 personas) y de algunas nacionalidades como la rusa (11\%) o la argelina (14\%); en este sentido, el "adel- 
gazamiento" de la pirámide poblacional de extranjeros empadronados en la provincia de Alicante 2014 con respecto a su predecesora (2013), aunque con carácter de provisionalidad, es evidente.

En la provincia de Alicante se da una elevada concentración de población extranjera en muy pocas nacionalidades, pues apenas 12 países acaparan el 74\% (2013); lo mismo ocurre en cuanto al número de ingresos de extranjeros en el HGUA, que tan solo los 6 primeros copan más de la mitad de los ingresos totales de la población extranjera, siendo la comunidad magrebí la que más uso hace de sus servicios; por tanto, aun siendo el colectivo de británicos el más numeroso en la provincia de Alicante, y a pesar de que se trata de un colectivo de edad avanzada, son superados en cuanto a número de ingresos hospitalarios en el HGUA por otros países como Marruecos, Colombia o Ecuador, debido fundamentalmente al número de ingresos de estos últimos en el área obstétrica, inclinando poderosamente la razón de sexo del total de la población nacida en el extranjero ingresada hacia el sexo femenino; pero si se obviasen los servicios de ginecología y obstetricia, la razón de sexo se inclinaría hacia los hombres $(52,8 \%$ de la población extranjera ingresada en el HGUA) que lideran los ingresos en más de la mitad de los servicios del HGUA (32 servicios), siendo mayor del 70\% en cardiología, cirugía cardíaca, cirugía pediátrica, hemodinámica y unidad de sangrantes digestivos, y entre el 60\%-70\% en otros 12 servicios.

Las patologías asociadas a la población extranjera hospitalizada son congruentes con la edad y presentan diferencias según se trate de extranjeros comunitarios (fundamentalmente enfermedades degenerativas, respiratorias y cardiovasculares) o extranjeros no comunita- rios (fundamentalmente embarazo, puerperio y alteraciones ginecológicas). Paradójicamente, el extranjero inmigrante joven es visto con enorme recelo, mientras que el extranjero residente es bien recibido en la mayoría de los casos. En términos de coste sanitario, las patologías que podrían asociarse a la inmigración son, en general, de muy bajo impacto económico (cabe recordar que un parto es un proceso fisiológico que culmina con el nacimiento de un bebé), mientras que los añosos residentes y turistas del centro y norte de Europa son portadores de enfermedades crónicas y degenerativas de alto coste farmacéutico y asistencial (de ahí el objetivo de la Generalitat Valenciana de recabar fondos compensatorios por atención a extranjeros, principalmente del ámbito de la Unión Europea).

La Ley 16/2012 de medidas urgentes para garantizar la sostenibilidad del Sistema Nacional de Salud y mejorar la calidad y seguridad de sus prestaciones habría dejado su impronta reflejada en un aumento de la edad media de los pacientes nacidos en el extranjero ingresados en el HGUA, que entre otras consecuencias, podría conllevar un aumento del gasto sanitario (los diferentes tipos de patologías suelen ir asociados a la edad) que difícilmente se podría compensar por el uso de las alternativas a la hospitalización convencional.

Independientemente de que las alternativas a la hospitalización convencional (Hospitales de Día, Cirugía sin Ingreso, Unidades de Hospitalización a Domicilio, Unidades de Corta Estancia, etc.) han tenido un gran impacto en el número de estancias hospitalarias, el descenso en cuanto a número de ingresos en el HGUA, tanto de población autóctona como de población nacida en el extranjero, quedaría estrechamente ligado a la disminución de población empadronada, descendiendo en 
cuatro años y medio (enero 2010-junio 2014) la población total atendida en el HGUA en 19.511 pacientes $(-6,7 \%)$ y la población nacida en el extranjero en 11.854 pacientes (-28\%).

Mención especial a ODUSALUD por velar por el cumplimiento del Derecho Universal a la protección de la Salud de la ciudadanía, sobretodo de los colectivos más vulnerables; posiblemente su registro de incidencias tan solo suponga la punta de un gran iceberg donde gran parte de los casos no pasarían a formar parte de la estadística sanitaria.

En la actualidad estamos asistiendo a la forja de un "nuevo" modelo de sociedad multicultural, cuya idiosincrasia va a generar toda una serie de necesidades y demandas en materia de salud que requerirá de un ingente esfuerzo multidisciplinar que sepa plasmar, desarrollar e implementar las estrategias idóneas capaces de reorientar debidamente las políticas sanitarias y de servicios sociales.

\section{BIBLIOGRAFÍA}

- Albert, R.M. (2007). El síndrome del desarraigo cultural. Murcia: Diego Marín.

- American Psychiatric Association (2014). Guía de consulta de los criterios diagnósticos del DSM-5 (Brevario). Madrid: Editorial Médica Panamericana.

- Asamblea General de las Naciones Unidas (1948). Declaración Universal de los Derechos Humanos de 10 de diciembre de 1948, París. Disponible en: http://www. cnrha.msssi.gob.es/bioetica/pdf/declaracion_Univ_Derechos_Humanos.pdf

- Barceló, M.V. (2014). Programa de investigación y difusión, Informe 2014. Aproximación estadística a la población extranjera en la provincia de Alicante. Astialicante. org. Secretariado Diocesano de Migración de OrihuelaAlicante. Disponible en: http://nadiesinfuturo.org/IMG/ pdf/INF--2014-COMPLETO.pdf

- Fernández, M.A. (2006a). Fenómenos migratorios y su influencia en los cuidados transculturales en el marco hospitalario. Cultura de los cuidados, 20, 62-68.

- Fernández, M.A. (2006b). Antropología de los cuidados. Estudio de los cuidados a pacientes extranjeros hospitalizados desde la perspectiva de la enfermería transcultural. Alicante: Consejo de Enfermería de la Comunitat Valenciana.

- Generalitat Valenciana (2013).Instrucción de la secretaría autonómica de sanidad, por la que se informa de la puesta en marcha del programa valenciano de protección de la salud. Valencia: Consellería de Sanitat. Disponible en: http://www.san.gva.es/documents/152919/169224/PROTECCION_SALUD.pdf

- Generalitat Valenciana (2014a). Data Warehouse SIP Sistema de información poblacional. Informe mensual junio 2014, Valencia: Consellería de Sanitat.

- Generalitat Valenciana (2014b). Sistema básico de Vigilancia Comunitat Valenciana. Casos y Tasas. Informe mensual Marzo 2014. Valencia: Direcció General de Salut Pública, Consellería de Sanitat.

- Generalitat Valenciana (2014). Epidemiología y Vigilancia de la Salud. Valencia: Dirección General de Salud Pública, Consellería de Sanitat. Disponible en: http://www. sp.san.gva.es/indexPortal.jsp?menuRaizPortal=SANMS5 0000\&Portal=EPIDEMIOLOGIA\&perfil=inst

- Gómez, C. (2005). Evolución de la población extranjera en la Comunidad Valenciana. Periodo 1998-2005. Una lectura amplia de indicadores actualizados. Observatorio Permanente de la Inmigración de la Universidad de Alicante. Alicante: Vicerrectorado de Extensión Universitaria, Alicante. Disponible en: http://www.ua.es/es/ estudios/seus/alacant/informes/evolinmigr98a05.pdf

- Gómez, C. (2014). Alicante se fractura. Informe del Observatorio Permanente de la Inmigración de la Universidad de Alicante. Vicerrectorado de Extensión Universitaria, Alicante.

- Hospital General de Alicante (HGUA). (2014). Hospital General Universitario de Alicante. Informes de alta codificados por la Unidad de Admisión y Documentación Clínica (UDCA) basados en el Conjunto Mínimo de Datos (CMBD). Alicante: UDCA

- Instituto Nacional de Estadística (INE). (2014a). Avance de la Estadística del Padrón Continuo a 1 de enero de 


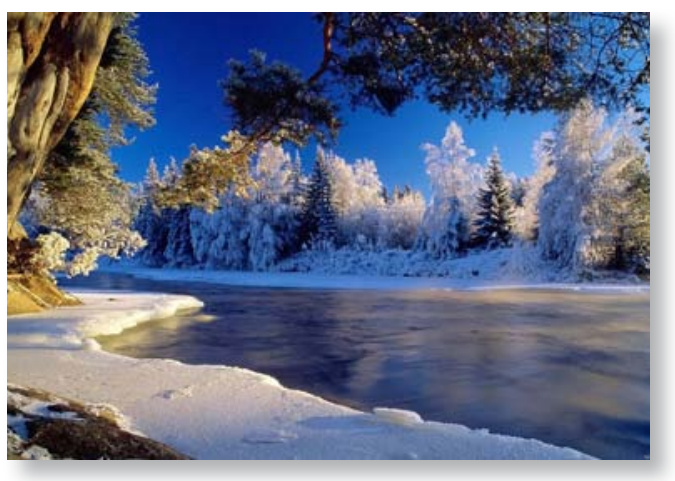

2014. Datos provisionales. Madrid: INE. Disponible en: http://www.ine.es/prensa/np838.pdf

- Instituto Nacional de Estadística (INE). Instituto Nacional de Estadística (2014b, 30 de junio) Cifras de población a 1 de enero de 2014. Estadística de Migraciones 2013. Datos provisionales. Madrid: INE. Disponible en: http://www.ine.es/prensa/np854.pdf

- Instituto Nacional de Estadística. INE.(2014). Inebase. Disponible en: www.ine.es

- Levante-EMV (2014). La nueva Ley de Salud crea un Comité de Pacientes para saber la opinión de los usuarios. Levante. El Mercantil Valenciano. Disponible en: http://www.levante-emv.com/comunitat-valenciana/2014/09/12/nueva-ley-salud-crea-comite/1160837. html

- Llamas, M. (2014). Británicos y alemanes abandonan España tras la histórica subida de impuestos. Libertad digital. Disponible en: http://www.libremercado.com/2014-05-23/britanicos-y-alemanes-abandonan-espana-tras-la-historica-subida-de-impuestos-1276519347/

- Mantecón, A., Huete, R., Estévez J.F. (2013). El impacto de la crisis económica sobre la movilidad internacional de los residentes extranjeros en la provincia de Alicante. Revista Internacional de Estudios Migratorios, 3: 155184.

- Melero L., Buades J., Díe L. et al. (2013) Informe anual 2013 sobre migraciones e integración. Una década de migraciones en España. Centro de Estudios para la Integración Social y Formación de Inmigrantes CeiMigra. Disponible en: http://www.ceimigra.net/observa-
torio/images/stories/luis_pdf/Informe_Anual_CeiMigra_2013_dp.pdf

- Membrado, J.C. (2014). La costa de los jubilados. La migración noreuropea de personas retiradas a la Costa Blanca. Revista Mètode 81. Disponible en:

http://metode.cat/es/Revistas/Monografics/Itinerancias/ La-costa-dels-jubilats

- ODUSALUD (2014). Observatorio del Derecho Universal a la Salud de la Comunitat Valenciana. Informe ${ }^{\circ} 8$ a 1 de julio 2014. Disponible en: https://docs.google. com/file/d/0B6xOMLiL6YCzTnFmcjlCbm45d0k/edit

- Oficina del Alto Comisionado de Naciones Unidas para los Derechos Humanos (1966). Pacto Internacional sobre Derechos Económicos, Sociales y Culturales, adoptado y abierto a la firma, ratificación y adhesión por la Asamblea General en su resolución 2200-A-(XXI), de 16 de diciembre de 1966. Disponible en: http:// www2.ohchr.org/spanish/law/cescr.htm

- Organización Mundial de la Salud (OMS). (1946). Constitución de la Organización Mundial de la Salud. Documentos Básicos, suplemento de la $45^{\mathrm{a}}$ edición, Octubre 2006. Disponible en: http://www.who.int/governance/ eb/who_constitution_sp.pdf

- Ortiz, F. et al. (2011). Estudio de personas extranjeras en SIP año 2010-2011. Generalitat Valenciana. SIP Servicio de Aseguramiento Sanitario DGECAP, Valencia: Consellería de Sanitat. Disponible en: http://www2.san.gva.es/docs/INFORME_EXTRANJEROS_SIP_2010.pdf

- Pascual, C. (2014) Los argelinos, fijos en Alicante. Diario Información. Disponible en: http://www.diarioinformacion.com/alicante/2014/08/10/argelinos-fijos-alicante/1534059.html

- SATSE (2014). SATSE informa: Mesa gral. Función Pública y convocatoria EIR 2014-2015. Documento divulgativo.

- Secretariado Diocesano de Migración de Orihuela-Alicante Asti-Alicante (2013). Aproximación estadística a la inmigración en Alicante. Programa de Investigación y Difusión. Informe CeiMigra 2013. Disponible en:

http://astialicante.org/wp-content/uploads/2013/05/ Aprox-Estad\%C3\%ADstica-Completa-2013.pdf 


\section{Fuentes primarias normativas}

- REGLAMENTO (CE) Nº 118/97 DEL CONSEJO de 2 de diciembre de 1996 por el que se modifica y actualiza el Reglamento (CEE) n 1408/71 relativo a la aplicación de los regímenes de Seguridad Social a los trabajadores por cuenta ajena, los trabajadores por cuenta propia y a los miembros de sus familias que se desplazan dentro de la Comunidad, y el Reglamento (CEE) n ${ }^{\circ} 574 / 72$ por el que se establecen las modalidades de aplicación del Reglamento $(\mathrm{CEE}) \mathrm{n}^{\circ} 1408 / 71$

Estatal

- CONSTITUCIÓN ESPAÑOLA. Aprobada por Las Cortes en sesiones plenarias del Congreso de los Diputados y del Senado celebradas el 31 de octubre de 1978. Ratificada por el pueblo español en referéndum de 6 de diciembre de 1978. Sancionada por S.M. el Rey ante Las Cortes el 27 de diciembre de 1978.

- LEY 14/1986, de 25 de abril, General de Sanidad.

- LEY ORGÁNICA 16/2003, de 28 de mayo, de cohesión y calidad del Sistema Nacional de Salud.

- LEY ORGÁNICA 4/2000, de 11 de enero, sobre derechos y libertades de los extranjeros en España y su integración social.

- LEY ORGÁNICA 8/2000, de 22 de diciembre, de reforma de la Ley Orgánica 4/2000, de 11 de Enero, sobre derechos y libertades de los extranjeros en España y su integración social.

- REAL DECRETO 1088/1989, de 8 de septiembre, por el que se extiende la cobertura de la asistencia sanitaria de la Seguridad Social a las personas sin recursos económicos suficientes.

- REAL DECRETO 2393/2004, de 30 de diciembre, por el que se aprueba el Reglamento de la Ley Orgánica 4/2000, de 11 de enero, sobre derechos y libertades de los extranjeros en España y su integración social.

- REAL DECRETO-LEY 16/2012, de 20 de abril, de medidas urgentes para garantizar la sostenibilidad del Sistema Nacional de Salud y mejorar la calidad y seguridad de sus prestaciones.

- REAL DECRETO LEY 1192/2012, de 3 de agosto, por el que se regula la condición de asegurado y de beneficiario a efectos de la asistencia sanitaria en España, con cargos a fondos públicos, a través del Sistema Nacional de Salud.

\section{Autonómica}

- LEY 6/2008, de 2 de junio, de la Generalitat, de Aseguramiento Sanitario del Sistema Sanitario Público de la Comunitat Valenciana

- DECRETO 1/2005, de 25 de Febrero, por el que se aprueba el texto refundido de la Ley de Tasas de la Generalitat, TR Ley de Tasas (Ejercicio 2014)

- DECRETO 126/1999, de 16 de agosto, del Gobierno Valenciano, por el que se crea el Sistema de Información Poblacional de la Conselleria de Sanidad

- DECRETO 26/2000, de 22 de febrero, por el que se establece el derecho a la asistencia sanitaria a ciudadanos extranjeros en la Comunidad Valenciana y se crea la Tarjeta Solidaria.

${ }^{1}$ Z60.3 "Acculturation Difficulty": pertenece al apartado "Otros problemas que pueden ser objeto de atención clínica”, que es una categoría con incertidumbre diagnóstica, debiéndose utilizar cuando una dificultad de adaptación a una cultura nueva (por ejemplo, relacionada con la emigración) es el objeto de la atención clínica o tiene algún impacto sobre el tratamiento o el pronóstico del individuo (American Psychiatric Association, 2014: 411)

${ }^{2}$ Este año se dobló holgadamente los datos de 2002.

${ }^{3}$ No se debe obviar el hecho de que los inmigrantes que querían regularizarse podían denunciar a los empresarios.

4 El director del Observatorio Permanente de la Inmigración, Carlos Gómez Gil constata que en la ciudad de Alicante la comunidad argelina sigue en constante aumento pese a la crisis, debido fundamentalmente a una relación histórica entre las costas de Argelia y la provincia de Alicante (con un ferry permanente entre Alicante-Orán) y al creciente malestar de esta población hacia su país y autoridades (Pascual C., 2014: 8).

${ }^{5}$ El RDL 16/2012, de 20 de Abril, de medidas urgentes para garantizar la sostenibilidad del SNS y mejorar la 
calidad y seguridad de sus prestaciones, en su art.1 modifica la ley 16/2003, de 28 de Mayo, de cohesión y calidad del SNS añadiendo el art.3 ter, con la siguiente redacción:

"Art.3 ter. Asistencia sanitaria en situaciones especiales.

Los extranjeros no registrados ni autorizados como residentes en España recibirán asistencia sanitaria en las siguientes modalidades:

* de urgencia por enfermedad grave o accidente, cualquiera que sea la causa, hasta la situación de alta médica.

* de asistencia al embarazo, parto y postparto..."

En cualquier caso, los extranjeros menores de dieciocho años recibirán asistencia sanitaria en las mismas condiciones que los españoles, y la competencia para el reconocimiento del derecho a la asistencia sanitaria corresponde a los Servicios Públicos de Salud de las Comunidades Autónomas, a través de protocolos establecidos

${ }^{6}$ En materia de aseguramiento sanitario, la nueva ley de Salud de la Comunitat Valenciana continuaría con la adaptación al Sistema de Información Poblacional (SIP) al nuevo modelo de aseguramiento del Sistema Nacional de Salud, así como la consolidación y completa integración funcional con la base de datos de tarjeta sanitaria única del Ministerio de Sanidad, Servicios Sociales e Igualdad.
${ }^{7}$ Los principales grupos de Aseguramiento que presenta la Ley 6/2008 son:

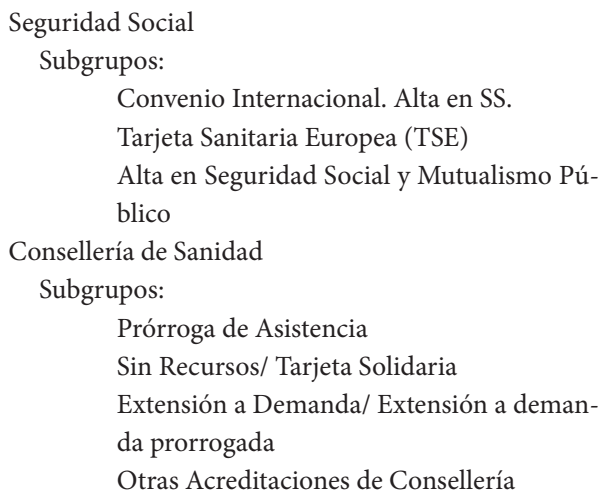

\section{Sin Asistencia en Centros Sanitarios Públicos} Subgrupos:

Acreditación Caducada
No Acreditados
Mutualismos Privados

En el año 2012 como consecuencia del RDL 16/2012, aparece una nueva categoría: "Asegurados Provisionales" al que pertenecen las personas, extranjeras o no, con cobertura provisional.

\begin{tabular}{llll}
\multicolumn{2}{c}{${ }^{8}$ Códigos/unidades de hospitalización* } & del HGUA: & \\
AGU & Agudos (Nefrología) & NER & Neurología \\
ALE & Alergia & NFL & Neurofisiología \\
CAI & Cardiología infantil & NRI & Neurología infantil \\
CAR & Cardiología & NRX & Neuroradiología \\
CCA & Cirugía cardíaca & OBS & Obstetricia \\
CGI & Cirugía pediátrica & OFI & Oftalmología infantil \\
CIM & Cuidados intermedios médicos & OFT & Oftalmología \\
CIR & Cirugía & ONC & Oncología \\
COT & Traumatología & ONI & Oncología infantil \\
CPI & Cirugía plástica infantil & ORI & Otorrinolaringología infantil \\
CPL & Cirugía plástica & ORL & Otorrinolaringología \\
CTO & Cirugía torácica & PED & Pediatría \\
CVA & Cirugía vascular & QUE & Quemados \\
DER & Dermatología & REA & Reanimación \\
DII & Medicina digestiva infantil & REU & Reumatología
\end{tabular}




\section{ᄃultura de las Cuidados}

$\begin{array}{llll}\text { ECI } & \text { Endocrinología infantil } & \text { ROD } & \text { Rodilla } \\ \text { ECR } & \text { Endocrinología } & \text { TRI } & \text { Traumatología infantil } \\ \text { FIV } & \text { Unidad de reproducción } & \text { UCE } & \text { Unidad de corta estancia } \\ \text { GIN } & \text { Ginecología } & \text { UCN } & \text { UCI neonatal } \\ \text { HEM } & \text { Hematología } & \text { UCP } & \text { UCI pediátrica } \\ \text { HMD } & \text { Hemodinámica } & \text { UDO } & \text { Unidad de dolor } \\ \text { IMP } & \text { Implantes (nefrología) } & \text { UEI } & \text { Unidad de enfermedades infecciosas } \\ \text { LAC } & \text { Lactantes } & \text { UHP } & \text { Unidad hepática } \\ \text { MDI } & \text { Medicina digestiva } & \text { UIC } & \text { Unidad de ictus } \\ \text { MIN } & \text { Medicina interna } & \text { UMI } & \text { Medicina intensiva } \\ \text { NCG } & \text { Neurocirugía } & \text { UPM } & \text { Unidad de patología mamaria } \\ \text { NCI } & \text { Neurocirugía infantil } & \text { URO } & \text { Urología } \\ \text { NEF } & \text { Nefrología } & \text { URQ } & \text { Unidad de raquis } \\ \text { NEM } & \text { Neumología } & \text { USD } & \text { Unidad de sangrantes digestivos } \\ \text { NEN } & \text { Neonatología } & & \end{array}$

${ }^{\star}$ Se alude a las unidades de hospitalización en las que se contabilizaron ingresos de pacientes nacidos en el extranjero

${ }^{9}$ Se pueden consultar los boletines epidemiológicos en la página web de la dirección General de Salud Pública en el área de Epidemiología de la Consellería de Sanitat de la Comunitat Valenciana

\section{TABLAS, FIGURAS Y GRÁFICOS.}

Tabla 1. Evolución de la población empadronada en España (2008-2013)

\begin{tabular}{l|r|r|r|r|r|r|r|r|} 
& \multicolumn{1}{c|}{$\mathbf{2 0 0 8}$} & \multicolumn{1}{c|}{$\mathbf{2 0 0 9}$} & $\mathbf{2 0 1 0}$ & $\mathbf{2 0 1 1}$ & $\mathbf{2 0 1 2}$ & $\mathbf{2 0 1 3}$ \\
\hline \hline Total población & 46.157 .822 & 46.745 .807 & 47.021 .031 & 47.190 .493 & 47.265 .321 & 47.129 .783 \\
Total población extranjera & 5.268 .762 & 5.648 .671 & 5.747 .734 & 5.751 .487 & 5.736 .258 & 5.546 .238 \\
Ciudadanos UE 27 & 2.102 .654 & 2.273 .226 & 2.350 .172 & 2.395 .358 & 2.443 .617 & 2.359 .371 \\
Resto extranjeros & 3.166 .108 & 3.375 .445 & 3.397 .562 & 3.356 .129 & 3.292 .641 & 3.186 .867 \\
\hline
\end{tabular}

Fuente: Elaboración propia a partir de la Estadística del Padrón Continuo a 1 de Enero de cada año. INE, Datos definitivos

Tabla 2.Población empadronada en España en el año 2014*y su variación interanual 2013-2014*

\begin{tabular}{|l|l|l|l|l|l|l|l|l|l|}
\hline \multicolumn{4}{|c|}{ Población empadronada } & \multicolumn{4}{c|}{ Variación interanual 2013-2014* } \\
\hline & $\begin{array}{l}\text { Población } \\
\text { total }\end{array}$ & $\begin{array}{l}\text { Total } \\
\text { población } \\
\text { extranjera }\end{array}$ & $\begin{array}{l}\text { Total } \\
\text { ciudadanos } \\
\text { UE 27** }\end{array}$ & $\begin{array}{l}\text { Total Resto } \\
\text { Extranjeros }\end{array}$ & $\begin{array}{l}\text { V.I: } \\
\text { Población } \\
\text { total }\end{array}$ & $\begin{array}{l}\text { V.I. Total } \\
\text { población } \\
\text { extranjera }\end{array}$ & $\begin{array}{l}\text { V.I. Total } \\
\text { ciudadanos } \\
\text { UE 27** }\end{array}$ & $\begin{array}{l}\text { V-I. Resto } \\
\text { extranjeros }\end{array}$ \\
\hline España & 46.725 .164 & 5.000 .258 & 2.047532 & 2.952 .726 & -404.619 & -545.980 & -311.839 & -234.141 \\
\hline
\end{tabular}

Fuente: Elaboración propia a partir de la Estadística del Padrón Continuo a 1 de Enero de 2013 y 2014(INE)

${ }^{*}$ Los datos del año 2014 son provisionales ${ }^{* *}$ Croacia se incorpora a la Unión Europea el 1 de Julio de 2014

Tabla 3. Evolución de la población extranjera empadronada en la Comunitat Valenciana

\begin{tabular}{lllllll} 
& $\mathbf{2 0 0 8}$ & $\mathbf{2 0 0 9}$ & $\mathbf{2 0 1 0}$ & $\mathbf{2 0 1 1}$ & $\mathbf{2 0 1 2}$ & $\mathbf{2 0 1 3}$ \\
\hline Total & 847.339 & 889.340 & 893.759 & 880.782 & 883.012 & 863.891 \\
Castellón & 106.125 & 111.221 & 112.265 & 111.402 & 111.598 & 108.443 \\
Valencia & 294.846 & 315.145 & 314.395 & 302.696 & 298.804 & 286.031 \\
Alicante & 446.368 & 462.974 & 467.099 & 466.684 & 472.610 & 469.417 \\
\hline
\end{tabular}

Fuente: Estadística del Padrón Continuo a 1 de Enero de cada año. INE, Datos definitivos 


\section{Cultura de las Cuidados}

Tabla 4. Población empadronada en la Comunitat Valenciana en el año 2014*y su variación interanual 2013-2014*

\begin{tabular}{|c|c|c|c|c|c|c|c|c|}
\hline & \multicolumn{4}{|c|}{ Población empadronada } & \multicolumn{4}{|c|}{ Variación interanual 2013-2014* } \\
\hline & $\begin{array}{l}\text { Población } \\
\text { total }\end{array}$ & $\begin{array}{l}\text { Total } \\
\text { población } \\
\text { extranjera }\end{array}$ & $\begin{array}{l}\text { Total } \\
\text { ciudadanos } \\
\text { UE } 27^{* *}\end{array}$ & $\begin{array}{l}\text { Total Resto } \\
\text { Extranjeros }\end{array}$ & $\begin{array}{l}\text { V.I: } \\
\text { Población } \\
\text { total }\end{array}$ & $\begin{array}{l}\text { V.I. Total } \\
\text { población } \\
\text { extranjera }\end{array}$ & $\begin{array}{l}\text { V.I. Total } \\
\text { ciudadanos } \\
\text { UE } 27^{* *}\end{array}$ & $\begin{array}{l}\text { V-I. Resto } \\
\text { extranjeros }\end{array}$ \\
\hline C. Valenciana & 4.995 .216 & 732.52 & 388.730 & 343.795 & -118.59 & -131.366 & -106.743 & -24.623 \\
\hline
\end{tabular}

Fuente: Elaboración propia a partir de la Estadística del Padrón Continuo a 1 de Enero de 2013 y 2014(INE)

*Los datos del año 2014 son provisionales ** Croacia se incorpora a la Unión Europea el 1 de Julio de 2013

Tabla 5.Población empadronada en la provincia de Alicante en el año 2014*y su variación interanual 2013-2014*

\begin{tabular}{|c|c|c|c|c|c|c|c|c|}
\hline & \multicolumn{4}{|c|}{ Población empadronada en la provincia de Alicante } & \multicolumn{4}{|c|}{ Variación interanual 2013-2014* } \\
\hline & $\begin{array}{l}\text { Población } \\
\text { total }\end{array}$ & $\begin{array}{l}\text { Total } \\
\text { población } \\
\text { extranjera }\end{array}$ & $\begin{array}{l}\text { Total } \\
\text { ciudadanos } \\
\text { UE } 27^{* *}\end{array}$ & $\begin{array}{l}\text { Total Resto } \\
\text { Extranjeros }\end{array}$ & $\begin{array}{l}\text { V.I: } \\
\text { Población } \\
\text { total }\end{array}$ & $\begin{array}{l}\text { V.I. Total } \\
\text { población } \\
\text { extranjera }\end{array}$ & $\begin{array}{l}\text { V.I. Total } \\
\text { ciudadanos } \\
\text { UE } 27^{* *}\end{array}$ & $\begin{array}{l}\text { V-I. Resto } \\
\text { extranjeros }\end{array}$ \\
\hline Alicante & $1.862 .35 \mathrm{~S}$ & 379.689 & 217.562 & 162.127 & -83.283 & -89.728 & -80.246 & 9.482 \\
\hline
\end{tabular}

Fuente: Elaboración propia a partir de la Estadística del Padrón Continuo a 1 de Enero de 2013 y 2014(INE)

*Los datos del año 2014 son provisionales ** Croacia se incorpora a la Unión Europea el 1 de Julio de 2013

Tabla 6. Población extranjera empadronada en la provincia de Alicante según zona de procedencia

\begin{tabular}{lrrrrrr} 
& $\mathbf{2 0 0 8}$ & $\mathbf{2 0 0 9}$ & $\mathbf{2 0 1 0}$ & $\mathbf{2 0 1 1}$ & $\mathbf{2 0 1 2}$ & $\mathbf{2 0 1 3}$ \\
\hline Total & 446.368 & 462.974 & 467.099 & 466.684 & 472.610 & 469.417 \\
\hline Unión Europea & 271.869 & 284.310 & 290.662 & 293.293 & 299.459 & 297.808 \\
Resto de Países Europeos & 35.264 & 36.345 & 37.011 & 38.011 & 40.263 & 41.603 \\
Países Africanos & 44.061 & 47.415 & 49.629 & 50.927 & 52.964 & 55.077 \\
Países Americanos & 84.897 & 83.428 & 77.451 & 70.796 & 64.637 & 58.729 \\
Países Asiáticos & 10.171 & 11.353 & 12.217 & 13.449 & 15.157 & 16.052 \\
Países de Oceanía y Apátridas & 106 & 123 & 129 & 120 & 130 & 148 \\
\hline
\end{tabular}

Fuente: Elaboración propia a partir de datos del INE a 1 de Enero de cada año. Datos definitivos

Tabla 7. Población extranjera empadronada en la provincia de Alicante según nacionalidad (doce primeras)

\begin{tabular}{|c|c|c|c|c|c|}
\hline & 2013 & & $2014^{*}$ & & \\
\hline Países & Población & $\%$ & Países & Población & $\%$ \\
\hline Total & 469.417 & 100 & & 379.689 & 100 \\
\hline Reino Unido & 130.541 & 28 & Reino Unido & 91.287 & 24 \\
\hline Marruecos & 36.692 & 8 & Marruecos & 36.513 & 10 \\
\hline Alemania & 35.916 & 8 & Rumanía & 30.204 & 8 \\
\hline Rumanía & 34.037 & 7 & Alemania & 21.441 & 6 \\
\hline Países Bajos & 17.192 & 4 & Federación Rusa & 14.429 & 4 \\
\hline Ecuador & 15.150 & 3 & Países Bajos & 13.649 & 4 \\
\hline Colombia & 13.826 & 3 & Argelia & 13.516 & 4 \\
\hline Bulgaria & 13.721 & 3 & Ecuador & 12.911 & 3 \\
\hline Federación Rusa & 12.969 & 3 & Colombia & 11.619 & 3 \\
\hline Argelia & 11.854 & 3 & Bulgaria & 11.475 & 3 \\
\hline Francia & 11.814 & 3 & Italia & 9.492 & 2 \\
\hline Italia & 11.393 & 2 & Francia & 9.051 & 2 \\
\hline Resto de países & 124.312 & 26 & Resto de países & 104.102 & 27 \\
\hline
\end{tabular}

Fuente: Elaboración propia a partir de la Estadística del Padrón Continuo a 1 de Enero de 2013 y 2014 (INE)

*Los datos del año 2014 son provisionales. Croacia se incorpora a la Unión Europea el 1 de Julio de 2013 
Figura 1. Población extranjera empadronada por edad (grupos quinquenales) y sexo en la provincia de Alicante.

Datos INE definitivos a 1 de Enero de 2013

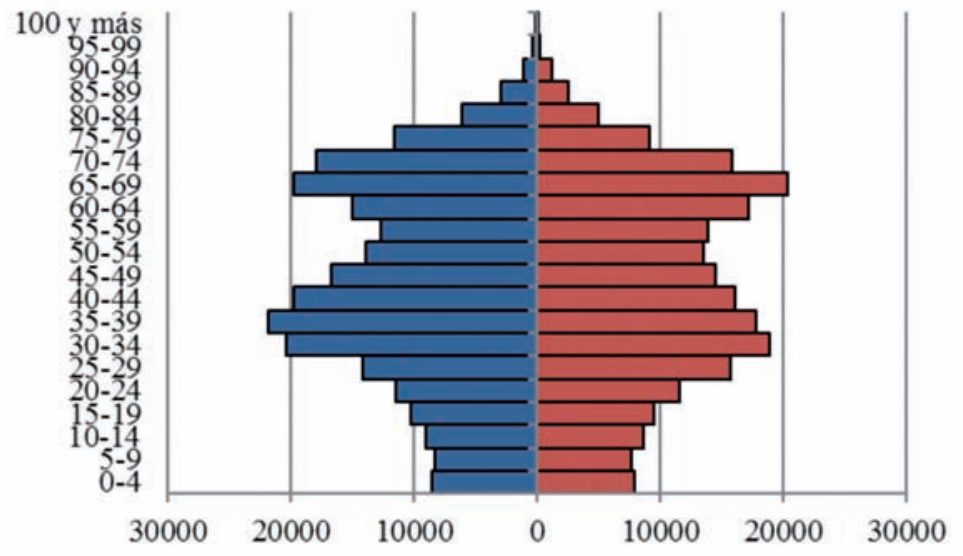

口Mujeres

口Hombres

Figura 2. Población extranjera empadronada por edad (grupos quinquenales) y sexo en la provincia de Alicante. Datos INE provisionales a 1 de Enero de 2014

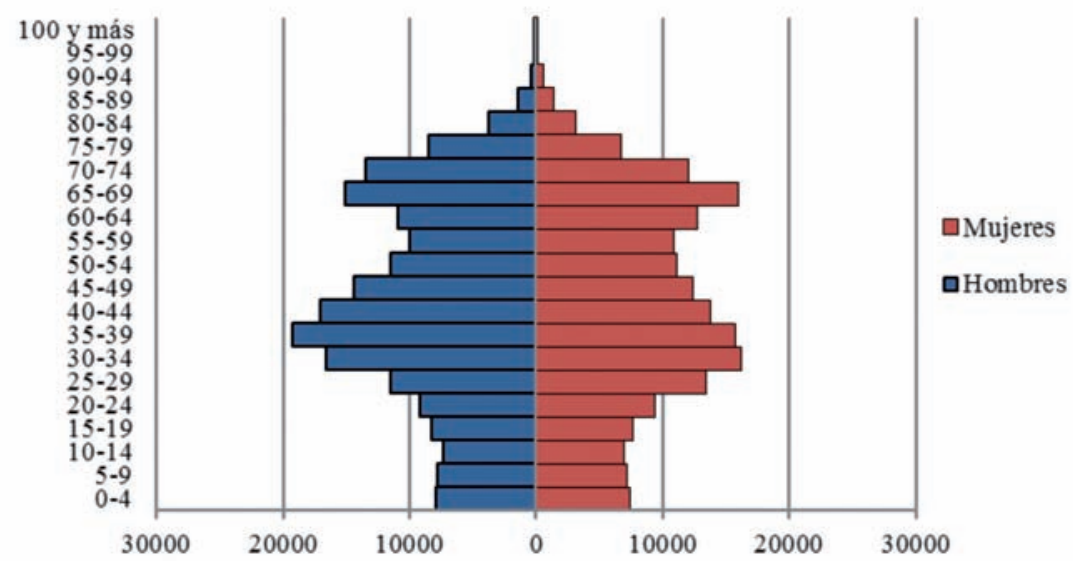


Tabla 8. Población extranjera empadronada por edad (grupos quinquenales) y sexo en la provincia de Alicante

\begin{tabular}{|c|c|c|c|c|c|c|}
\hline \multirow[b]{2}{*}{ Edad } & \multicolumn{3}{|c|}{ Año 2013} & \multicolumn{3}{|c|}{ Año 2014* } \\
\hline & Total & Hombres & Mujeres & Total & Hombres & Mujeres \\
\hline $0-4$ & 16.484 & 8.535 & 7.949 & 15.352 & 7.911 & 7441 \\
\hline $5-9$ & 16.006 & 8.306 & 7.700 & 14.936 & 7.755 & 7181 \\
\hline $10-14$ & 17.702 & 9.021 & 8.681 & 14.354 & 7.387 & 6967 \\
\hline 15-19 & 19.854 & 10.271 & 9.583 & 15.987 & 8.319 & 7668 \\
\hline $20-24$ & 23.093 & 11.447 & 11.646 & 18.585 & 9.242 & 9343 \\
\hline 25-29 & 29.866 & 14.087 & 15.779 & 24.918 & 11.541 & 13377 \\
\hline 30-34 & 39.326 & 20.358 & 18.968 & 32.877 & 16.628 & 16249 \\
\hline 35-39 & 39.623 & 21.764 & 17.859 & 34.987 & 19.203 & 15784 \\
\hline $40-44$ & 35.583 & 19.750 & 16.103 & 30.903 & 17.091 & 13812 \\
\hline $45-49$ & 31.227 & 16.630 & 14.597 & 26.747 & 14.350 & 12397 \\
\hline $50-54$ & 27.380 & 13.864 & 13.516 & 22.679 & 11.548 & 11131 \\
\hline 55-59 & 26.541 & 12.619 & 13.922 & 20.865 & 10.016 & 10849 \\
\hline $60-64$ & 32.154 & 14.963 & 17.191 & 23.619 & 10.892 & 12727 \\
\hline $65-69$ & 40.147 & 19.734 & 20.413 & 31.073 & 15.151 & 15922 \\
\hline $70-74$ & 33.875 & 17.927 & 15.948 & 25.548 & 13.467 & 12081 \\
\hline 75-79 & 20.818 & 11.623 & 9.195 & 15.191 & 8.526 & 6665 \\
\hline $80-84$ & 11.015 & 6.023 & 4.992 & 6.906 & 3.762 & 3144 \\
\hline $85-89$ & 5.512 & 2.904 & 2.608 & 2.936 & 1.489 & 1447 \\
\hline $90-94$ & 2.329 & 1.110 & 1.219 & 1.008 & 419 & 589 \\
\hline 95-99 & 593 & 275 & 318 & 188 & 65 & 123 \\
\hline 100 y más & 19 & 5 & 14 & 30 & 11 & 19 \\
\hline Total & 469.417 & 241.216 & 228.201 & 379.689 & 194.773 & 184.916 \\
\hline
\end{tabular}

Fuente: INE. Estadística del Padrón Contínuo a 1 de Enero de cada año *Datos provisionales.

Tabla 9. Variación interanual 2013-2014 de la población extranjera empadronada por edad (grupos quinquenales) y sexo en la provincia de Alicante

\begin{tabular}{|c|c|c|c|c|c|c|}
\hline Edad & Total & $\%$ & Hombres & $\%$ & Mujeres & $\%$ \\
\hline $0-4$ & -1.132 & 1,3 & -624 & 1,3 & -508 & 1,2 \\
\hline $5-9$ & -1.070 & 1,2 & -551 & 1,2 & -519 & 1,2 \\
\hline $10-14$ & -3.348 & 3,7 & -1.634 & 3,5 & -1.714 & 4,0 \\
\hline $15-19$ & -3.867 & 4,3 & -1.952 & 4,2 & -1.915 & 4,4 \\
\hline $20-24$ & -4.508 & 5,0 & -2.205 & 4,7 & -2.303 & 5,3 \\
\hline 25-29 & -4.948 & 5,5 & -2.546 & 5,5 & -2.402 & 5,5 \\
\hline $30-34$ & -6.449 & 7,2 & -3.730 & 8,0 & -2.719 & 6,3 \\
\hline $35-39$ & -4.636 & 5,2 & -2.561 & 5,5 & -2.075 & 4,8 \\
\hline $40-44$ & -4.680 & 5,2 & -2.659 & 5,7 & -2.291 & 5,3 \\
\hline $45-49$ & -4.480 & 5,0 & -2.280 & 4,9 & -2.200 & 5,1 \\
\hline $50-54$ & -4.701 & 5,2 & -2.316 & 5,0 & -2.385 & 5,5 \\
\hline 55-59 & -5.676 & 6,3 & -2.603 & 5,6 & -3.073 & 7,1 \\
\hline $60-64$ & -8.535 & 9,5 & -4.071 & 8,8 & -4.464 & 10,3 \\
\hline 65-69 & -9.074 & 10,1 & -4.583 & 9,9 & -4.491 & 10,4 \\
\hline $70-74$ & -8.327 & 9,3 & -4.460 & 9,6 & -3.867 & 8,9 \\
\hline $75-79$ & -5.627 & 6,3 & -3.097 & 6,7 & -2.530 & 5,8 \\
\hline $80-84$ & -4.109 & 4,6 & -2.261 & 4,9 & -1.848 & 4,3 \\
\hline $85-89$ & -2.576 & 2,9 & -1.415 & 3,0 & -1.161 & 2,7 \\
\hline $90-94$ & -1.321 & 1,5 & -691 & 1,5 & -630 & 1,5 \\
\hline 95-99 & -405 & 0,5 & -210 & 0,5 & -195 & 0,5 \\
\hline 100 y más & 11 & 0,0 & 6 & 0,0 & 5 & 0,0 \\
\hline Total & -89.728 & 100,0 & -46.443 & 100,0 & -43.285 & 100,0 \\
\hline
\end{tabular}

Fuente: Elaboración propia a partir de datos INE. Datos provisionales 
Tabla 9. Variación interanual 2013-2014 de la población extranjera empadronada por edad (grupos quinquenales) y sexo en la provincia de Alicante

\begin{tabular}{lrrrrrr}
\hline \multicolumn{1}{c}{ Edad } & \multicolumn{1}{c}{ Total } & \% & Hombres & $\%$ & Mujeres & $\%$ \\
$\mathbf{0 - 4}$ & -1.132 & 1,3 & -624 & 1,3 & -508 & 1,2 \\
$\mathbf{5 - 9}$ & -1.070 & 1,2 & -551 & 1,2 & -519 & 1,2 \\
$\mathbf{1 0 - 1 4}$ & -3.348 & 3,7 & -1.634 & 3,5 & -1.714 & 4,0 \\
$\mathbf{1 5 - 1 9}$ & -3.867 & 4,3 & -1.952 & 4,2 & -1.915 & 4,4 \\
$\mathbf{2 0 - 2 4}$ & -4.508 & 5,0 & -2.205 & 4,7 & -2.303 & 5,3 \\
$\mathbf{2 5 - 2 9}$ & -4.948 & 5,5 & -2.546 & 5,5 & -2.402 & 5,5 \\
$\mathbf{3 0 - 3 4}$ & -6.449 & 7,2 & -3.730 & 8,0 & -2.719 & 6,3 \\
$\mathbf{3 5 - 3 9}$ & -4.636 & 5,2 & -2.561 & 5,5 & -2.075 & 4,8 \\
$\mathbf{4 0 - 4 4}$ & -4.680 & 5,2 & -2.659 & 5,7 & -2.291 & 5,3 \\
$\mathbf{4 5 - 4 9}$ & -4.480 & 5,0 & -2.280 & 4,9 & -2.200 & 5,1 \\
$\mathbf{5 0 - 5 4}$ & -4.701 & 5,2 & -2.316 & 5,0 & -2.385 & 5,5 \\
$\mathbf{5 5 - 5 9}$ & -5.676 & 6,3 & -2.603 & 5,6 & -3.073 & 7,1 \\
$\mathbf{6 0 - 6 4}$ & -8.535 & 9,5 & -4.071 & 8,8 & -4.464 & 10,3 \\
$\mathbf{6 5 - 6 9}$ & -9.074 & 10,1 & -4.583 & 9,9 & -4.491 & 10,4 \\
$\mathbf{7 0 - 7 4}$ & -8.327 & 9,3 & -4.460 & 9,6 & -3.867 & 8,9 \\
$\mathbf{7 5 - 7 9}$ & -5.627 & 6,3 & -3.097 & 6,7 & -2.530 & 5,8 \\
$\mathbf{8 0 - 8 4}$ & -4.109 & 4,6 & -2.261 & 4,9 & -1.848 & 4,3 \\
$\mathbf{8 5 - 8 9}$ & -2.576 & 2,9 & -1.415 & 3,0 & -1.161 & 2,7 \\
$\mathbf{9 0 - 9 4}$ & -1.321 & 1,5 & -691 & 1,5 & -630 & 1,5 \\
$\mathbf{9 5 - 9 9}$ & -405 & 0,5 & -210 & 0,5 & -195 & 0,5 \\
$\mathbf{1 0 0}$ y más & 11 & 0,0 & 6,0 & 0,0 & 5 & 0,0 \\
Total & -89.728 & 100,0 & -46.443 & 100,0 & -43.285 & 100,0 \\
\hline
\end{tabular}

Fuente: Elaboración propia a partir de datos INE. Datos provisionales

Tabla 10. Evolución de la población total y extranjera registradas en SIP en la Comunitat Valenciana (2005-2012)

\begin{tabular}{lrrr}
\hline Años & Pobl. total & Pobl. Extranjera & \% pobl.extranjera \\
$\mathbf{2 0 0 5}$ & 4.954 .419 & 518.001 & 10,46 \\
$\mathbf{2 0 0 6}$ & 5.129 .972 & 598.289 & 11,66 \\
$\mathbf{2 0 0 7}$ & 5.179 .279 & 717.229 & 13,85 \\
$\mathbf{2 0 0 8}$ & 5.423 .428 & 868.206 & 16,01 \\
$\mathbf{2 0 0 9}$ & 5.779 .634 & 979.495 & 16,95 \\
$\mathbf{2 0 1 0}$ & 5.695 .705 & 976.273 & 17,14 \\
$\mathbf{2 0 1 1}$ & 5.282 .199 & 868.476 & 16,44 \\
$\mathbf{2 0 1 2}$ & 5.144 .277 & 774.268 & 15,05 \\
\hline
\end{tabular}

Fuente: Extracto de la tabla $\mathrm{n}^{\circ} 8$ del Informe CeiMigra, 2013, p. 44, basado en los Informes SIP de la Consellería de Sanitat (2005-2012)

Tabla 11. Modalidades de acreditación de la asistencia sanitaria. Evolución de los ciudadanos extranjeros en SIP en el primer semestre de 2014

\begin{tabular}{|c|c|c|c|c|c|c|c|}
\hline $\begin{array}{c}\text { Modalidades aseguramiento } \\
\text { (año 2014) }\end{array}$ & Enero & Febrero & Marzo & Abril & Mayo & Junio & $\begin{array}{l}\text { Variación } \\
\text { Ene-Jun }\end{array}$ \\
\hline Totales & 765.979 & 766.697 & 767.298 & 766.788 & 767.199 & 740.262 & -25.717 \\
\hline Asegurados INSS-TSE & 581.360 & 582.594 & 582.835 & 582.711 & 582.940 & 583.936 & 2.576 \\
\hline Cobertura SNS-Consellería & 19.610 & 20.332 & 20.896 & 21.346 & 21.486 & 21.656 & 2.046 \\
\hline Asegurados provisionales & 11.805 & 11.709 & 11.552 & 11.479 & 11.391 & 11.051 & -754 \\
\hline $\begin{array}{l}\text { Sin cobertura. Mutualismo } \\
\text { privado-No acreditado }\end{array}$ & 153.204 & 152.062 & 152.015 & 151.252 & 151.312 & 123.619 & -29.585 \\
\hline
\end{tabular}

Información de ciudadanos extranjeros incluidos en SIP: Empadronados y no empadronados (estancia $>1$ mes) en la C. Valenciana Fuente: Generalitat Valenciana, Sistema de Información Poblacional; extracto de la página 40 del informe mensual, Junio 2014 
Tabla 12. Porcentaje de población nacida en el extranjero ingresada en el HGUA según zona de procedencia

\begin{tabular}{lrrrr}
\hline & $\mathbf{2 0 1 1}$ & $\mathbf{2 0 1 2}$ & $\mathbf{2 0 1 3}$ & $\mathbf{( 2 0 1 1 - 2 0 1 3 )}$ \\
\hline Unión Europea & 32,33 & 32,87 & 31,03 & 32,10 \\
Resto de Países Europeos & 4,49 & 3,58 & 7,09 & 5,01 \\
Países Africanos & 23,56 & 22,77 & 23,09 & 23,15 \\
Países Americanos & 35,55 & 36,19 & 37,20 & 36,28 \\
Países Asiáticos & 3,70 & 4,35 & 2,17 & 3,44 \\
Países de Oceanía y Apátridas & 0,03 & 0,03 & 0 & 0,02 \\
\hline Fuente: Elaboración propia a partir de los informes de alta codificados por la Unidad de Admisión y \\
Documentación Clínica HGUA
\end{tabular}

Tabla 13. Población nacida en el extranjero ingresada en el HGUA desde el 1 de Enero de 2011 hasta el 31 de Diciembre de 2013 y sus respectivas variaciones interanuales
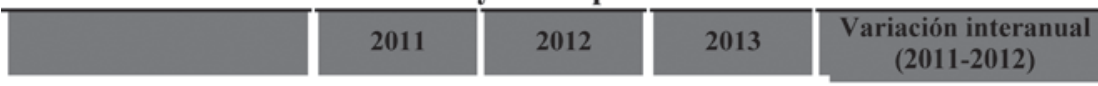

Hombres

1.315

1.312

1.241 (2011-2012)

Mujeres

1.979

1.788

1.708

$-3$

Variación interanua

Total extranjeros

3.294

3.100

2.949

$-191$

Fuente: elaboración propia a partir de los informes de alta codificados por la Unidad de Admisión y Documentación Clínica HGUA

Tabla 14 Población nacida en el extranjero ingresada en el HGUA desde el 1 de Enero hasta el 30 de Juni (años 2013 y 2014). Variación intersemestral.

\begin{tabular}{|c|c|c|c|c|c|c|c|c|c|}
\hline & \multicolumn{3}{|c|}{ Enero-Junio 2013} & \multicolumn{3}{|c|}{ Enero-Junio 2014} & \multicolumn{3}{|c|}{ Variación intersemestral } \\
\hline & $\bar{M}$ & $\mathrm{H}$ & Total & $\bar{M}$ & $\overline{\mathrm{H}}$ & Total & $\bar{M}$ & $\mathrm{H}$ & Total \\
\hline Población autóctona & 6.970 & 7.385 & 14.355 & 7.374 & 7.399 & 14.773 & 404 & 14 & 41 \\
\hline Extranjeros & 845 & 665 & 1.510 & 870 & 589 & 1.459 & 25 & -76 & -8 \\
\hline No codificados & 46 & 64 & 110 & 44 & 61 & 105 & -2 & -3 & \\
\hline Total general & 7.861 & 8.114 & 15.975 & 8.288 & 8.049 & 16.337 & 447 & -65 & $3 t$ \\
\hline
\end{tabular}

Fuente: Elaboración propia a partir de los informes de alta codificados por la Unidad de Admisión y Documentación Clíniı HGIIA

\begin{tabular}{|c|c|c|c|c|c|c|c|}
\hline \multicolumn{8}{|c|}{$\begin{array}{l}\text { Tabla 15. } N^{\circ} \text { pacientes nacidos en el extranjero ingresados en el HGUA, desglosados por códigos }{ }^{1} \\
\text { de servicios médicos }\end{array}$} \\
\hline OBS & 2.020 & HMD & 199 & UPM & 72 & ECI & 8 \\
\hline CIR & 665 & MDI & 199 & QUE & 70 & LAC & 8 \\
\hline CAR & 433 & URO & 192 & PED & 69 & NRI & 6 \\
\hline UCE & 425 & MIN & 190 & UMI & 69 & UDO & 6 \\
\hline NEM & 402 & CPL & 182 & ECR & 63 & CIM & 5 \\
\hline GIN & 321 & ONC & 182 & CCA & 60 & NEN & 5 \\
\hline COT & 319 & СTO & 147 & URQ & 54 & UCP & 4 \\
\hline NCG & 312 & CGI & 129 & REU & 50 & OFI & 3 \\
\hline UHP & 296 & ROD & 116 & ORI & 45 & AGU & 3 \\
\hline UEI & 253 & UIC & 99 & IMP & 32 & ALE & 2 \\
\hline ORL & 244 & ONI & 96 & NCI & 25 & DER & 2 \\
\hline HEM & 220 & TRI & 94 & DII & 24 & CAI & 1 \\
\hline CVA & 218 & OFT & 90 & REA & 21 & FIV & 1 \\
\hline NEF & 212 & NRX & 78 & USD & 15 & UCN & 1 \\
\hline NER & 203 & NFL & 73 & CPI & 10 & & \\
\hline
\end{tabular}

Fuente: Elaboración propia a partir de los informes de alta codificados por la Unidad de $\overline{\text { Admisión y }}$ Documentación Clínica HGUA 


\begin{tabular}{lr} 
Tabla 16. Primeros doce países con \\
mayor número de ingresos totales en \\
el HGUA (2011-2013) \\
\hline Marruecos & 1.244 \\
Colombia & 839 \\
Ecuador & 728 \\
Reino Unido & 697 \\
Francia & 644 \\
Argentina & 611 \\
Argelia & 494 \\
Rumanía & 426 \\
Alemania & 219 \\
Federación Rusa & 205 \\
Bolivia & 193 \\
Cuba & 176 \\
Resto de países & 2.867 \\
\hline Suma & 9.343 \\
\hline Fuente: Elaboración propia a partir de los \\
informes de alta codificados por la Unidad \\
de Admisión y Documentación Clínica \\
HGUA
\end{tabular}

Tabla 17. Variación intersemestral desde el 1 Enero al 30 Junio (años 2013 y 2014) de la población nacida en el extranjero ingresada en el HGUA, según países

\begin{tabular}{lrrr}
\hline Países & $\begin{array}{l}\text { Variación intersemestral } \\
\text { Ene-Jun 2013-2014 }\end{array}$ & \multicolumn{2}{c}{ \% Variación intersemestral } \\
Marruecos & -15 & $-4,3$ \\
Colombia & -33 & $-12,3$ \\
Argentina & 35 & 14,5 \\
Francia & -4 & $-1,9$ \\
Reino Unido & -12 & $-5,8$ \\
Ecuador & -29 & $-15,3$ \\
Argelia & -8 & $-4,8$ \\
Rumanía & 20 & 14,9 \\
Federación Rusa & 0 & 0,0 \\
Alemania & -6 & $-8,8$ \\
Cuba & 1 & 1,5 \\
Bolivia & -1 & $-1,7$ \\
\hline Fuente: Elaboración propia a partir de los informes de alta codificados por la Unidad de \\
Admisión y Documentación Clínica HGUA
\end{tabular}

Tabla 18. Población nacida en el extranjero atendida en el servicio de Obstetricia del HGUA desde el 1 de Enero de 2011 hasta el 31 de Diciembre de 2013 y sus respectivas variaciones interanuales

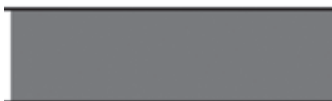

España

Nacidas en el extranjero

No codificados

Total general

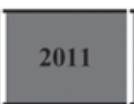

$$
2.629
$$

753

24

3.406

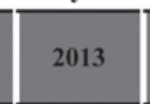

2.171

584

16

2.771

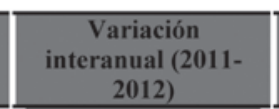

7.294

2.020

61

9.375

$-135$

$-70$

$-3$

$-208$

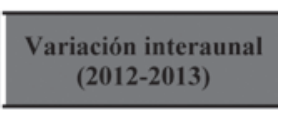

$-323$

$-99$

$-5$

$-427$ HGUA 
Tabla 19 Variación intersemestral (1 Enero-30 Junio 2013 y 2014)

de la población ingresada en la unidad de obstetricia del HGUA

\begin{tabular}{lrrr}
\hline & Ene-Jun & Ene-Jun & \multicolumn{2}{l}{$\begin{array}{l}\text { Variación } \\
\text { intersemestral }\end{array}$} \\
\hline Población autóctona & 1.093 & 899 & -194 \\
Extranjeros & 286 & 242 & -44 \\
No codificados & 6 & 8 & 2 \\
Total general & 1.385 & 1.149 & -236 \\
\hline Fuente: Elaboración propia a partir de los informes de alta codificados por la \\
Unidad de Admisión y Documentación Clínica HGUA
\end{tabular}

Unidad de Admisión y Documentación Clínica HGUA

Tabla 20. Diagnósticos principales de los pacientes nacidos en el extranjero ingresados en el HGUA (20112013) atendiendo a GRDs, y su coste (en euros)

\begin{tabular}{lrrl}
\hline Códigos & \multicolumn{1}{l}{ N $^{\circ}$} & & \\
GRDs & casos & \multicolumn{1}{c}{ Importe } & Descripción GRDs \\
\hline 373 & 688 & $1.708,46$ & Parto sin complicaciones \\
372 & 463 & $2.104,52$ & Parto con complicaciones \\
371 & 213 & $2.794,59$ & Cesárea sin complicaciones \\
073 & 194 & $1.744,24$ & Otros diagnósticos de oído, naríz, boca y garganta $>17$ \\
381 & 146 & $1.621,42$ & Aborto con dilatación y legrado, aspiración o histerotomía \\
370 & 123 & $3.449,21$ & Cesárea, con complicaciones \\
494 & 104 & $3.149,15$ & Colecistectomía laparosc. sin explorac. conducto biliar sin cc \\
359 & 97 & $4.190,79$ & Procedim. sobre útero y añejos por carcinoma in situ y proc. no malignos, sin cc \\
321 & 96 & $1.766,39$ & Infecciones de riñón y tracto urinario en menores de 18 años \\
883 & 94 & $3.181,33$ & Apendicectomía laparoscópica \\
541 & 85 & $5.000,25$ & Neumonía simple y otros trast. respirat. exc. bronquitis y asma con cc mayor \\
886 & 85 & $2.210,82$ & Otros diagnósticos anteparto sin procedimiento quirúrgico \\
125 & 81 & $2.552,45$ & Trast. circulatorios excepto IAM, con cateterismo sin diagn. complejo \\
410 & 81 & $4.120,36$ & Quimioterapia \\
112 & 79 & $3.691,49$ & Procedimientos cardiovasculares percutáneos, sin IAM, insuf. Cardíaca o shock \\
\hline
\end{tabular}

Fuente: Elaboración propia a partir de los informes de alta codificados por la Unidad de Admisión y Documentación Clínica HGUA y Ley de Tasas (Ejercicio 2014)

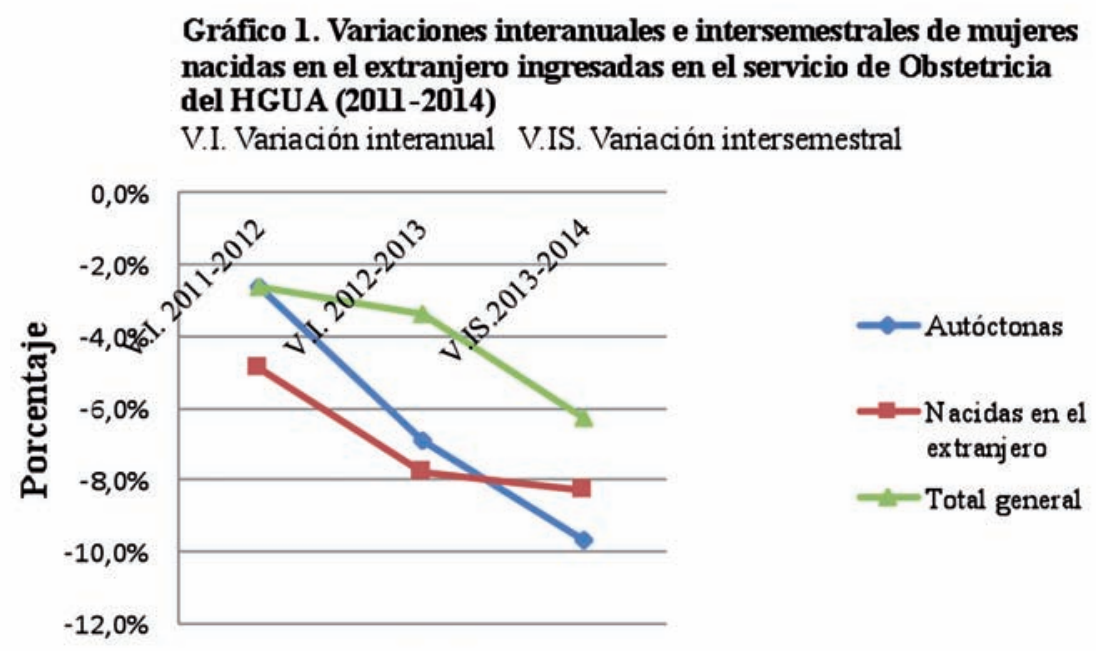

